\title{
Corridors of Mei-Yu-Season Rainfall over Eastern China
}

\author{
PeiYing Guan, GuiXing Chen, WenXin Zeng, AND Qian LiU \\ School of Atmospheric Sciences and Guangdong Province Key Laboratory for Climate Change and Natural Disaster Studies, \\ Sun Yat-sen University, Guangzhou, and Southern Marine Science and Engineering Guangdong Laboratory (Zhuhai), \\ Zhuhai, China
}

(Manuscript received 26 August 2019, in final form 14 November 2019)

\begin{abstract}
Successive mesoscale convective systems may develop for several days during the mei-yu season (JuneJuly) over eastern China. They can yield excessive rainfall in a narrow latitudinal band (called a corridor), causing severe floods. The climatology of rainfall corridors and related environmental factors are examined using $20 \mathrm{yr}$ of satellite rainfall and atmospheric data. A total of 93 corridors are observed over eastern China, with maximum occurrence at $27^{\circ}-31^{\circ} \mathrm{N}$. They typically last $2-3$ days, but some persist $\geq 4$ days, with an extreme event lasting 11 days. These multiday convective episodes exhibit primary and secondary peaks in the morning and afternoon, respectively, with a diurnal cycle that is in contrast to other afternoon-peak rain events. On average, the corridors occur in $\sim 23 \%$ days of the mei-yu season, but they can contribute $\sim 51 \%$ of the total rainfall. They also vary with years and explain $\sim 70 \%$ of the interannual variance of mei-yu-season rainfall. Composite analyses show that most corridors develop along zonally oriented quasi-stationary mei-yu fronts over central China where monsoon southwesterlies converge with northerly anomalies from the midlatitudes. The monsoon flow accelerates at $\sim 0200$ LST and forms a regional wind maximum or low-level jet over South China, which induces moisture flux convergence in morning-peak corridors. The nocturnal acceleration is less evident for afternoon-peak corridors. The mei-yu front and monsoon southwesterlies also influence the corridor's duration, which is regulated by a dipole of geopotential anomalies, with positive in the tropics and negative in the midlatitudes. The dipole expresses a joint influence of the blocking patterns in midlatitudes and the El Niño-related anomalous high over the western Pacific Ocean, and the dipole's intensity explains well the interannual variations of the corridors.
\end{abstract}

\section{Introduction}

Persistent extreme precipitation events are one of the most devastating disasters over eastern China and the central U.S. plains during the warm season (e.g., Tang et al. 2006; Groisman et al. 2012; Chen and Zhai 2013; Feng et al. 2016, 2019). A kind of extreme event is caused by mesoscale convective systems (MCSs) that successively develop in several days and propagate eastward following a similar path (Carbone et al. 2002; Wang et al. 2004; Jiang et al. 2006). The accumulated rainfall can be excessive in a narrow latitudinal zone (called a corridor), in a robust contrast to nearby areas that experience below-normal rainfall (Tuttle and Davis 2006, hereinafter TD06). Such rainfall corridors can cause severe flooding and contribute greatly to regional rainfall budget (TD06; Fritsch et al. 1986; Trier et al.

Corresponding author: Dr. Guixing Chen, chenguixing@mail. sysu.edu.cn
2014; Chen et al. 2017). Therefore, understanding the nature and environmental factors of rainfall corridors has valuable implications for regional climate studies, extreme rainfall prediction, disaster prevention, and water resource management.

It has been recognized that heavy rainfall bands prefer to occur at the east lee of huge terrain during the warm season (e.g., Carbone et al. 2002; Wang et al. 2004). Rainfall corridors over the central U.S. plains consist of $68 \%$ of the mesoscale rain events migrating from the eastern slope of the Rocky Mountains (TD06). The successive MCSs can cause a heavy rainfall band with a longitudinal extent greater than $1000 \mathrm{~km}$ but a narrow latitudinal width of $\sim 300 \mathrm{~km}$ (Trier et al. 2006, 2014). Similar east-west-oriented bands of heavy rainfall are also observed over East Asia (Fig. 1a) (e.g., Ninomiya 1991; Ding and Chan 2005; Geng and Yamada 2007; Sun and Zhang 2012). Chen et al. (2017) found that a 5-day corridor event with a rainfall amount of approximately 150 $400 \mathrm{~mm}$ occurred over eastern China, which extended up 
(a)

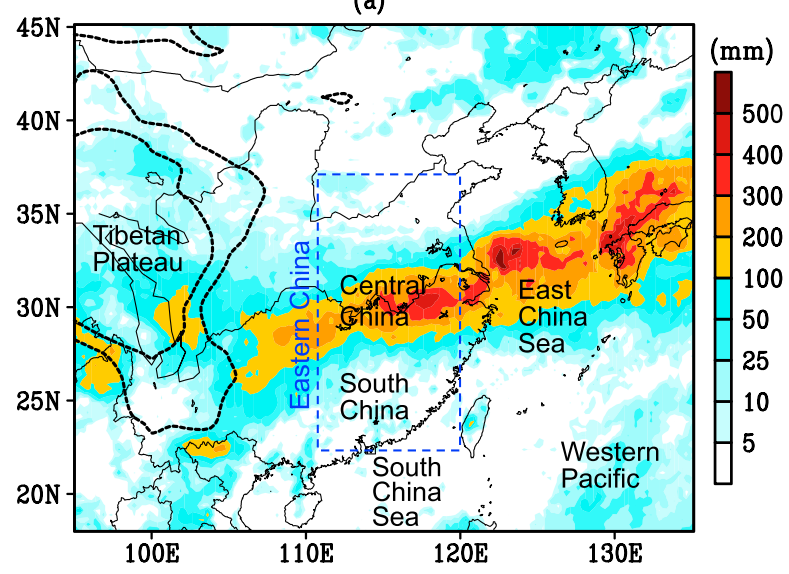

(b)

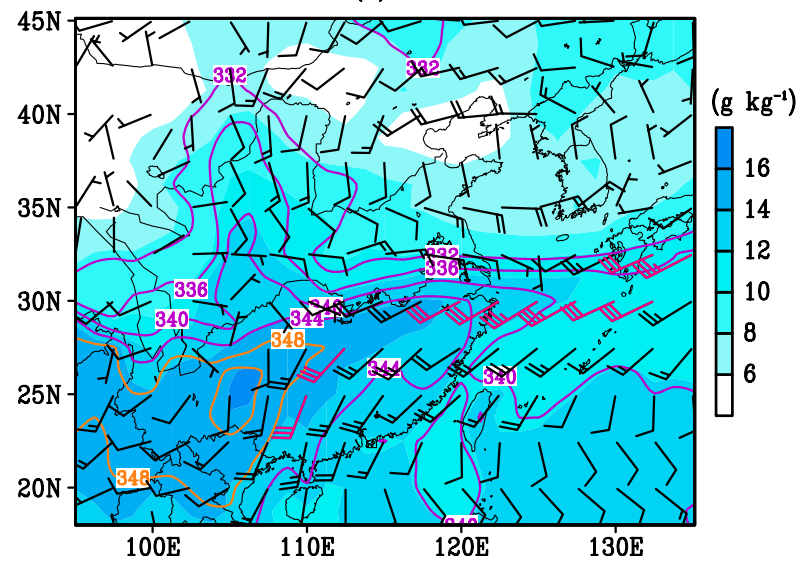

FIG. 1. A rainfall corridor that occurred over East Asia during 25-30 Jun 1999: (a) Accumulated rainfall amount derived from TRMM 3B42. The black dashed lines denote the elevations of 1500 and $3000 \mathrm{~m}$. The blue dashed box denotes eastern China $\left(112^{\circ}-\right.$ $120^{\circ} \mathrm{E}, 23^{\circ}-37^{\circ} \mathrm{N}$ ). (b) The 850 -hPa specific humidity (shading), equivalent potential temperature (contours), and horizontal winds (short and long barbs denote 2 and $4 \mathrm{~m} \mathrm{~s}^{-1}$, respectively).

to $\sim 1300 \mathrm{~km}$ with a narrow width of $\sim 160 \mathrm{~km}$. The rainfall corridors include multiday convective episodes that successively develop in the mei-yu frontal zone for several days (Zhang et al. 2014a, 2018). In the past, the climatology of rainfall corridors over eastern China has been unavailable because of a lack of consistent long-archive radar data that resolve mesoscale rainfall events. As the archive of high-quality satellite rainfall reaches up to $\sim 20$ years, it provides a viable approach for documenting the rainfall corridors over eastern China (Fig. 1a).

Previous studies have noted that warm-season rainfall over both the eastern China plains and the central U.S. plains exhibits pronounced diurnal variations (e.g., Wallace 1975; Yu et al. 2007; Carbone and Tuttle 2008; Chen et al. 2009; Zhang et al. 2014b). The rainfall over the central U.S. plains usually maximizes from midnight to predawn, with an eastward-delayed phase due to the propagating rain events (TD06; Trier et al. 2014). Over the eastern China plains, summer rainfall exhibits a feature of double peaks in the morning and afternoon (Xu and Zipser 2011; Yuan et al. 2013; Yu et al. 2014). The morning peak may be related to the mei-yu rainband that tends to intensify since late night (Geng and Yamada 2007; Sun and Zhang 2012). Case studies revealed that the morning-peak rainfall over eastern China can be produced by several nocturnal MCSs that develop concurrently along the mei-yu front, with their eastward propagation being slower than that over the central U.S. plains (Chen et al. 2012, 2017). So far, the rainfall corridors over eastern China remain to be clarified in a climatological manner with regard to their diurnal cycles, influence on regional rainfall budget, and differences from their U.S. counterparts.

Many studies have investigated the atmospheric conditions associated with the diurnal variations and persistence of precipitation systems over the central United States and eastern China (e.g., Trier et al. 2006; Carbone and Tuttle 2008; Chen et al. 2009, 2013; Yin et al. 2009; Sun and Zhang 2012; Xue et al. 2018). Augustine and Caracena (1994) attributed the nighttime long-lived MCSs to nocturnal low-level jets (NLLJs) and frontogenesis. TD06 noted that the repeated occurrences of NLLJs in the central U.S. plains are important to support successive MCSs in a corridor by enhancing convergence and transporting moisture. Over eastern China, monsoon southwesterlies regularly accelerate at night and form several mesoscale NLLJs extending downstream (Chen et al. 2017; Xue et al. 2018; Fu et al. 2019). At the northern terminus of NLLJs (Fig. 1b), a train of MCSs (Fig. 1a) develops along the mei-yu frontal zone where low-level ascent, moisture transport, convective instability, and meiyu frontogenesis are evident (Chen et al. 2000, 2017). The long-lived moist convection may be also regulated by other large-scale environmental factors such as the divergence of the upper-level jet (ULJ), meridional flow with blocking pattern in midlatitudes, and the activities of the western Pacific subtropical high (WPSH) and South Asian high (SAH) (Wang 1992; W.-C. Wang et al. 2000; Ninomiya and Shibagaki 2007; Sampe and Xie 2010; Ren et al. 2013; Chen and Zhai 2014; Y. Chen et al. 2019). For instance, the enhanced WPSH can regulate the regional diurnal variation of low-level winds for several days. This downscaling process of WPSH results in the repeated occurrence of mesoscale rain events downstream of the low-level winds (Zeng et al. 2019). The large-scale circulations and regional forcings seem to jointly affect the rainfall corridors. To date, our knowledge on the rainfall corridors over eastern China has been based on case studies; a climate analysis is called for to clarify the associated environmental conditions. 
This study uses a long archive of satellite rainfall to reveal the climatology of rainfall corridors over eastern China in the mei-yu season (June-July) from 1998 to 2017. We first examine the properties of corridors, with emphasis on their occurrence, rainfall pattern, diurnal variation, and the differences from their U.S. counterparts. Then, we clarify the large-scale atmospheric conditions that govern the diurnal cycle and duration of corridors. We will address how the key environmental factors regulate corridors through statistical analysis. The rest of the article is organized as follows. In section 2, we describe the dataset and objective methods for identifying corridors. In section 3 , we investigate the properties of corridors and their contribution to rainfall budget. The atmospheric conditions associated with the diurnal cycles of corridors are analyzed in section 4 . Section 5 illustrates the large-scale conditions that govern the duration and interannual variations of rainfall corridors. Conclusions and discussion are offered in section 6.

\section{Data and methods}

\section{a. Satellite-derived rainfall and atmospheric reanalysis data}

To describe the rainfall footprint of MCSs, we use satellite product from the Tropical Rainfall Measuring Mission (TRMM 3B42 V7). TRMM offers rainfall estimates at $0.25^{\circ}$ grids at 3-hourly interval, which resolves well the mesoscale to synoptic-scale features of rainfall activities (Huffman et al. 2007). The satellite rainfall has been calibrated by monthly rain gauge records, and thus it gives a reliable estimation of seasonal rainfall amount, which helps us examine the impacts of corridors on rainfall budget. TRMM is also found to capture the spatial patterns of rainfall diurnal cycles when compared with hourly gauge observations, particularly in event-based sense (Zhou et al. 2008; Chen et al. 2016; G. Chen et al. 2018). It performs well in resolving the overall distribution and diurnal cycle of a rainfall corridor over eastern China (Chen et al. 2017). Using a long archive of TRMM from 1998 to 2017, we may derive a 20-yr climatology of rainfall corridors. For verification, we also use the satellite rainfall from the Climate Prediction Center morphing technique (CMORPH) at higher resolutions of $8 \mathrm{~km}$ and $30 \mathrm{~min}$ (Joyce et al. 2004). Results show that CMORPH yields results that are consistent with TRMM with regard to the identification of corridor events. Given that TRMM performs marginally better for resolving the interannual variations of rainfall (G. Chen et al. 2018), we mainly present the analyses using TRMM data.

To show atmospheric conditions, we use the 55-Year Japanese Reanalysis (JRA-55), a kind of new-generation global analysis dataset with advanced model physics and data assimilation scheme (Kobayashi et al. 2015). JRA-55 provides 6-hourly reanalysis at a spatial resolution of $1.25^{\circ}$, which has been found to faithfully resolve the diurnal cycles over eastern China (Chen et al. 2014). In particular, it represents well the diurnal variations of rainfall, indicating a good capture of the physical processes associated with rainfall systems. With a vertical interval of $25 \mathrm{hPa}$ below $750 \mathrm{hPa}$, JRA-55 is also thought to depict well the lower-tropospheric conditions associated with the rainfall corridors. To examine the oceanic conditions, we also use the daily anomaly of Optimum Interpolation SST (OISST) provided by NOAA at a spatial resolution of $0.25^{\circ}$ (Reynolds et al. 2007). In this study, four synoptic hours $(1400,2000,0200$, and 0800 $\mathrm{LST}$; LST $=\mathrm{UTC}+8 \mathrm{~h}$ ) denote the early afternoon, early evening, late night, and the next morning, respectively.

\section{b. Identification of MCS centroids and rainfall corridors}

TD06 shows that a rainfall corridor consists of two or more rain events (MCSs) that develop in a same narrow band for more than one day. The rain events should somewhat overlap with the previous ones and drift slowly in the north-south direction. In this study, we employ a similar objective method to identify the mesoscale rain events (centroids) associated with corridors in eastern China (the blue dashed box shown in Fig. 1a) during the mei-yu season (June-July) of 1998-2017. The parameters in the method are modified because satellite-derived rainfall is used in our study. We focus on the rainfall footprint of MCSs that includes both convective and stratiform rain stages. Thus, the centroids may have a duration longer than those MCSs in previous studies that mainly describe convective stage using cloud-top temperature of blackbody (e.g., Maddox 1980; Augustine and Howard 1991; Laing and Fritsch 1997). Rain-gauge observations reveal that the rain events with a long duration of $\geq 6 \mathrm{~h}$ can account for a major part of the summer rainfall amount over eastern China (Yu et al. 2007). The scale analysis also noted that meso- $\alpha$-scale rain events with a horizontal extent of $\geq 150 \mathrm{~km}$ are dominant in eastern China (Chen et al. 2013). Therefore, we choose to apply a duration of $\geq 6 \mathrm{~h}$ and a latitudinal width of $\geq 1^{\circ}$ to identify the centroids in the zonally averaged plane.

The procedures for identifying rainfall corridors are detailed as follows. The TRMM rainfall is averaged over $112^{\circ}-120^{\circ} \mathrm{E}$ and stored in a two-dimensional (latitudetime) format (e.g., Fig. 2a). Similar to TD06, the centroids are enclosed by the zonally averaged rain rate above $1 \mathrm{~mm} \mathrm{~h}^{-1}$ in Fig. 2a. Each centroid should have a continuous rainy area at least eight grids (i.e., about $6 \mathrm{~h}$ long and $1^{\circ}$ wide). The central latitude of each centroid is estimated as the mean latitude of rainy area weighted by the square of rain rate. Those centroids that satisfy the three criteria 
(a)

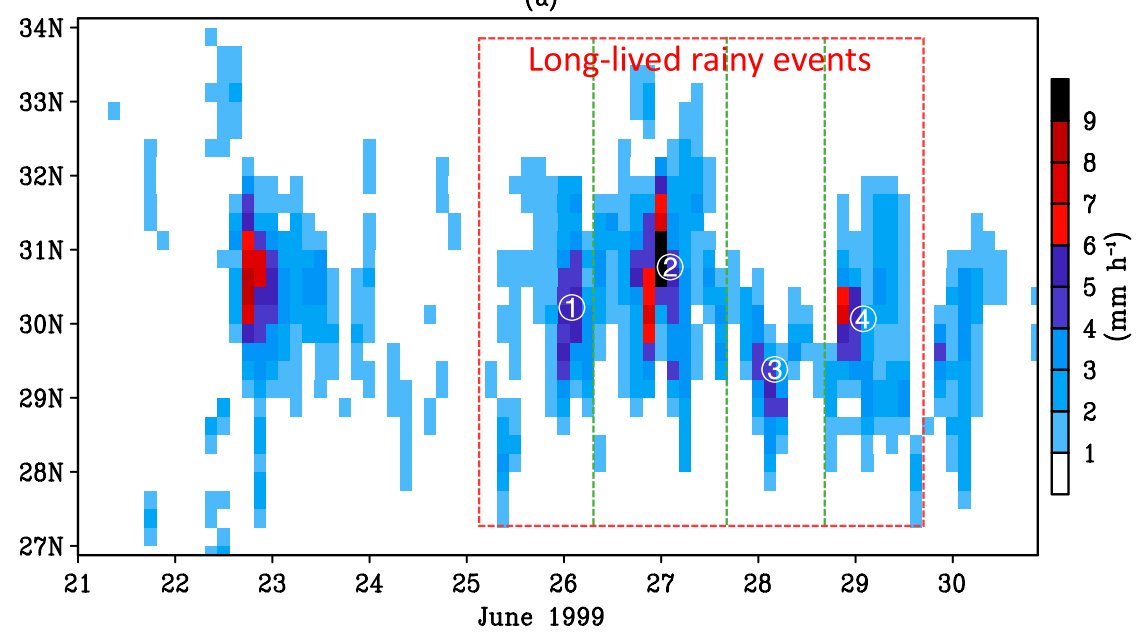

(b)

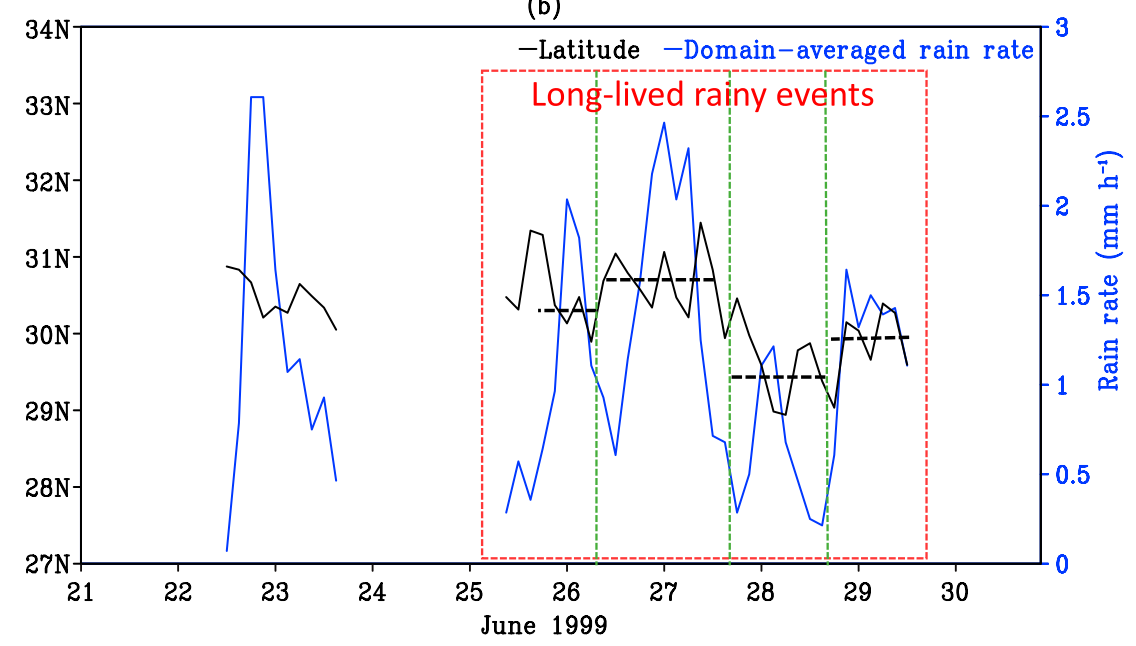

FIG. 2. Convective mesoscale centroids of the corridor in Fig. 1: (a) Latitude-time diagram of rain rate averaged at $112^{\circ}-120^{\circ} \mathrm{E}$. Four centroids are marked during $25-30$ Jun 1999 . (b) The 3-hourly variations of domain-averaged rain rate $\left(27^{\circ}-34^{\circ} \mathrm{N}, 112^{\circ}-120^{\circ} \mathrm{E}\right)$ and latitude of the rainfall. The black dashed lines show the central latitudes of the four centroids. The red dashed boxes denote long-lived rainy events, and the green dashed lines denote the borderlines between centroids.

below will be regarded as a rainfall corridor. First, the adjacent centroids can overlap with each other in a 30-h window, with a rate exceeding $25 \%$ of the area of the smaller one. The overlapped area is estimated by the artificial shift of centroids along the time axis of the latitudetime plane. Second, the distance between the central latitudes of adjacent centroids is less than $3^{\circ}$. Third, the duration of a corridor can last for at least 2 days, which is measured from the beginning of the first centroid to the end of the last one. The latitude of the corridor is estimated by the averaged latitudes of all involved centroids.

A few rainfall events with connected rainy grids may have a long duration of more than 1 day, which actually includes several centroids (Fig. 2a). To distinguish individual centroids within such events, we conduct additional handling as follows. We examine the 3-hourly variations of domain-averaged rain rate and latitude of the rainfall (Fig. 2b). Thus, we can objectively separate individual centroids from each other based on the hours of minimum rain rate (blue solid) and the sudden change of the rainfall's latitude (black dashes), which likely results from different MCSs. Then, these centroids are handled by the abovementioned procedures to obtain their central latitudes and ratio of overlapped area, as well as the information of the corridor. The results can be verified using CMORPH data at higher resolutions (figure not shown). Because TRMM performs marginally better for resolving the interannual variations of rainfall (G. Chen et al. 2018), we mainly present the analysis results using TRMM data. 

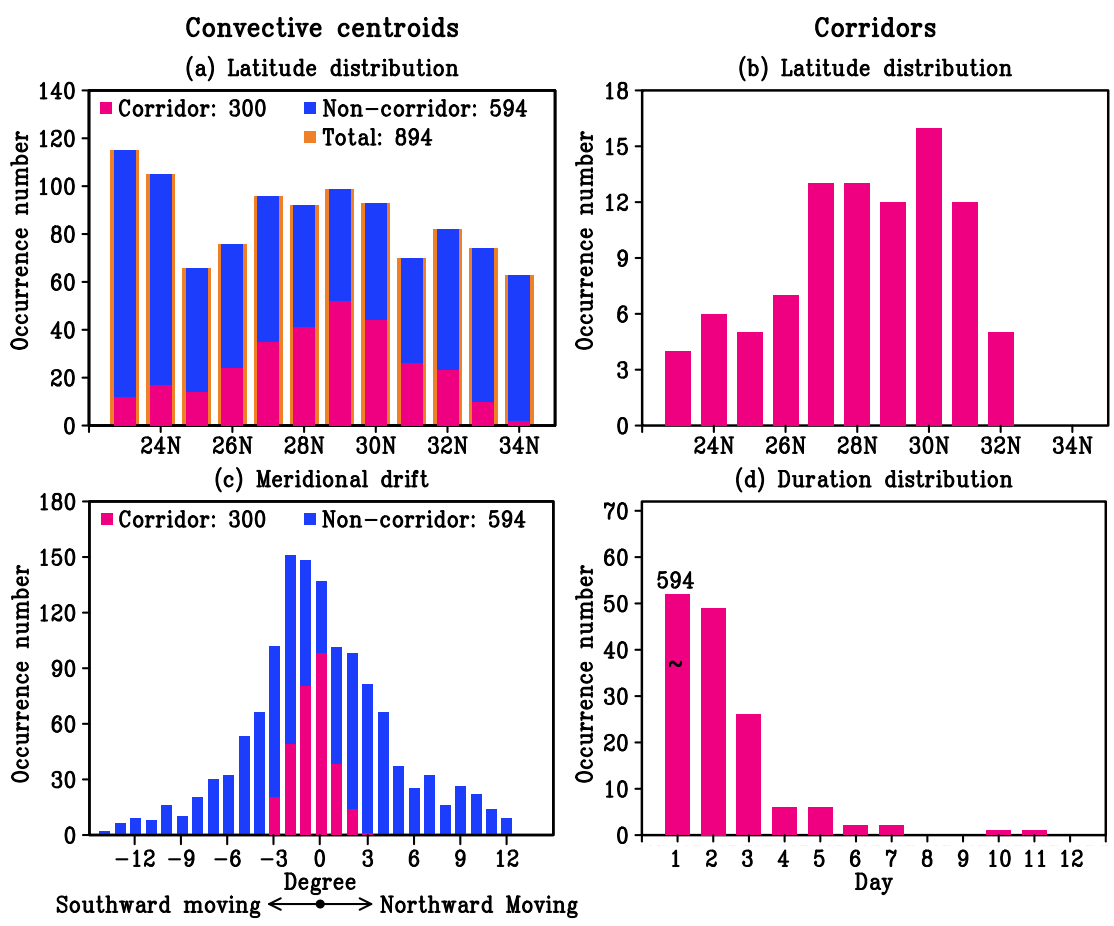

FIG. 3. (a) Latitude distribution and (c) meridional drift of the convective centroids. Only the centroids at $23^{\circ}-34^{\circ} \mathrm{N}$ are shown in (a) because they possibly belong to the corridor events. (b) Latitude distribution and (d) duration distribution of the corridors. The 1-day-duration events in (d) are categorized as non-corridor-type centroids.

\section{Occurrence of rainfall corridors and their impacts on rainfall budget}

\section{a. Statistics of the corridor occurrence}

Figure 3a shows that a total of 894 MCS centroids are observed over eastern China during the mei-yu season of 1998-2017, with $\sim 45$ for one year on average. The occurrence of convective centroids is most active at the South China coast and central China, which is consistent with the climatology of MCSs (e.g., Yang et al. 2015). About onethird of the centroids occur in a successive manner with slow north-south drifts, forming a total of 93 corridors. These corridor-type centroids have a maximum occurrence at $29^{\circ} \mathrm{N}$, where about one-half of the centroids belong to rainfall corridors. Figure $3 \mathrm{~b}$ shows that rainfall corridors have a well-defined maximum of occurrence at $27^{\circ}-31^{\circ} \mathrm{N}$ where $71 \%$ of corridor events are observed. There are $\sim 13$ corridor events occurring at each latitude, in contrast to an average of $\sim 5$ events at other latitudes. Such a concentrated occurrence of corridors may be associated with the mei-yu front at central China (Ding and Chan 2005). As a comparison, at the east lee of the Tibetan Plateau $\left(105^{\circ}-112^{\circ} \mathrm{E}\right)$, the corridors have fewer occurrences (60 events) in which rainfall systems develop along the north-south-oriented foothills (figure not shown). Similarly, the convective centroids are confined to the corridors in the central U.S. plains rather than in the foothills of the Rocky Mountains (TD06; Trier et al. 2006, 2014). We also note that the preferred latitudes of corridors over eastern China are relatively southern, as compared with those $\left(38^{\circ}-43^{\circ} \mathrm{N}\right)$ in the United States.

The slow north-south drift and prolonged duration are the key features of rainfall corridors, by which accumulated precipitation can be substantial in confined areas (TD06). Figure 3c shows that the daily drift of convective centroids in the corridor is limited to $\pm 3^{\circ}$, corresponding to the quasi-stationary feature of rainfall corridors. About $32.7 \%$ (98) of the corridor-type centroids are nearly stationary without north-south drifting. Another 49.7\% (149) tend to move southward (from $-1^{\circ}$ to $-3^{\circ}$ ), whereas only $17.6 \%$ (53) tend to move northward $\left(1^{\circ}-3^{\circ}\right)$. Such southward tendency is probably due to a southward-moving front or a surface cold pool (Bao and Zhang 2013; Luo et al. 2014; Trier et al. 2014; Xue et al. 2018). Figure 3d shows that 75 events among 93 corridors lasted for 2-3 days, 12 events last for 4-5 days, and 6 events last for more than 6 days, with an extreme event up to 11 days. These events cumulatively contribute to the number of rainy days about 176,54 , and 47 days, respectively. The shortlived corridors seem to have the largest occurrence, but the medium-lived and the long-lived corridors also 

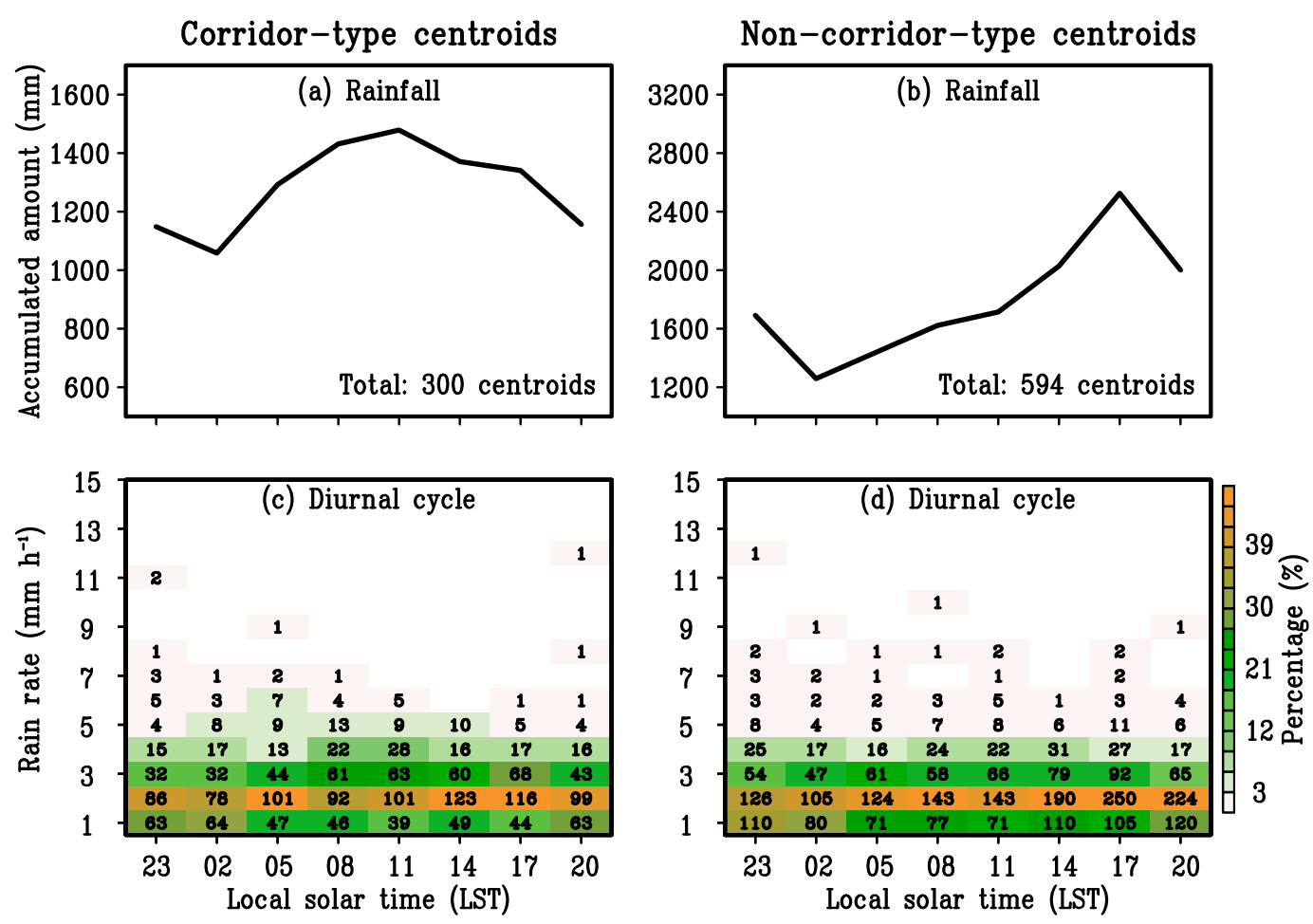

FIG. 4. (a),(b) Accumulated rainfall amount and (c),(d) rain-rate histograms of 300 (left) corridor-type centroids in their affecting zone $\left(3^{\circ}\right.$ latitude wide and $5^{\circ}$ longitude long) and (right) non-corridor-type centroids.

have a considerable occurrence, which are similar to those in the central U.S. plains (TD06; Trier et al. 2014).

\section{b. Diurnal cycle of the corridor occurrence and associated rainfall}

Diurnal variation of rainfall is one of the key features over eastern China (e.g., Yu et al. 2007; Chen et al. 2009). In this section, we examine the diurnal cycle of rainfall corridors. Figure 4 a shows that the accumulated rainfall of corridors increases by more than $40 \%$ after 0200 LST and reached its maximum of $1479 \mathrm{~mm}$ at 1100 LST. This morning-peak feature is also seen in the observed mei-yu rainband (Geng and Yamada 2007). The corridor-induced rainfall remains substantial during 1400-1700 LST and decays to a low value after 2000 LST (Fig. 4a). Such a primary morning peak of rainfall with a secondary feature in afternoon is different from those corridors with a single nocturnal peak over the central U.S. plains. Further analysis shows that the number of convective centroids also increases by $\sim 21 \%$ from 0200 to 1400 LST (figure not shown), which seems to partly contribute to the diurnal variation in rainfall amount. Another part of the rainfall diurnal variation is attributed to the relatively high rainfall intensity during 0800-1100 LST, when the occurrence of mean rain rate above $3 \mathrm{~mm} \mathrm{~h}^{-1}$ increases remarkably (Fig. 4c). Figure 4b shows that the non-corridor-type rainfall is suppressed during 0200-1100 LST and instead exhibits a significant peak at 1700 LST. This afternoonpeak rainfall coincides with its occurrence in terms of both diurnal phase and amplitude (not shown), while the mean rain rate is usually smaller than $3 \mathrm{~mm} \mathrm{~h}^{-1}$ and exhibits less diurnal variation (Fig. 4d). Such a large difference of rainfall diurnal cycles between corridor-type and non-corridortype centroids is analogous to that between long-duration and short-duration rain events observed over eastern China (Yu et al. 2007; Yuan et al. 2010; Wu et al. 2018).

We further examine the diurnal cycles of rainfall in the corridor and adjacent regions. To facilitate the composite analysis, the corridor events are artificially shifted to $30^{\circ} \mathrm{N}$, a latitude with the highest corridor occurrence (Fig. 3b). Figure 5a shows that obvious rainfall is concentrated in a narrow band of $300-400 \mathrm{~km}$ wide, with a mean rain rate above $1 \mathrm{~mm} \mathrm{~h}^{-1}$ from predawn to early afternoon. The rainfall is suppressed in the nearby areas outside the corridor and exhibits an afternoon peak along with a morning minimum. Such a robust contrast between corridor and nearby areas suggests that largescale conditions favorable for corridor may suppress precipitation to the south and north (Trier et al. 2014), which is discussed in sections 4 and 5. In contrast, the rainfall by non-corridor-type centroids instead maximizes in the afternoon over both centroids and nearby areas, which might be associated with the thermal convection induced by strong surface heating (Fig. 5b). 
Corridor-type centroids

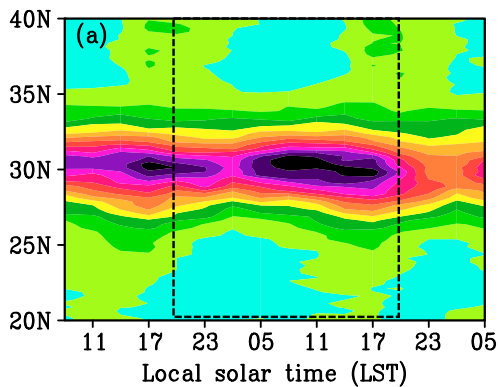

Non-corridor-type centroids
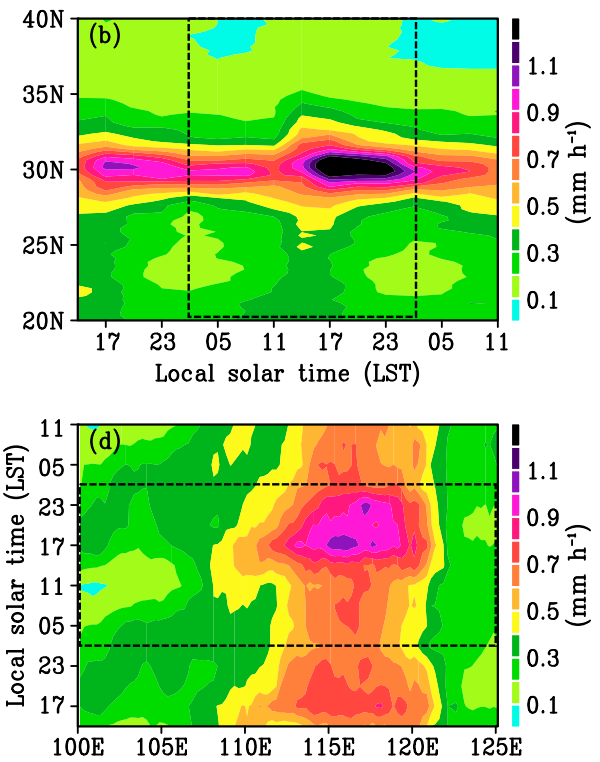

FIG. 5. (a) Latitude-time diagram of rain rate (shading) averaged at $112^{\circ}-120^{\circ} \mathrm{E}$ in corridor days. (c) Longitude-time diagram of rain rate averaged at $27^{\circ}-31^{\circ} \mathrm{N}$ in corridor days. All of the corridor days are composited in a 24 -h window as denoted by the dashed box. For a broader view, rainfall in the hours prior to/after each corridor day is shown as well. (b),(d) As in (a) and (c), but for non-corridor-type days. The convective centroids have been artificially shifted to $30^{\circ} \mathrm{N}$.

Figure $5 \mathrm{c}$ shows that there are three rainfall centers occuring at $100^{\circ}-103^{\circ} \mathrm{E}, 105^{\circ}-111^{\circ} \mathrm{E}$, and $112^{\circ}-120^{\circ} \mathrm{E}$ (corridor zone), respectively. The rainfall is obvious from midnight to morning with an eastward-delayed peak hour, which is similar to the climate state but at a stronger rain rate (Chen et al. 2010, 2012). These spatial modes are thus particularly active during the days with corridors. The atmospheric conditions that favor corridors over central China also help the rainfall in the zones of the same latitudes, although they suppress the rainfall to the northern/southern areas, indicating a large-scale hydrological impact. In contrast, rainfall by non-corridor-type events seems to develop locally in central China, with little change in the western/eastern areas (Fig. 5d).

\section{c. Impacts of corridors on the rainfall budget and interannual variations}

Because corridor events can produce substantial rainfall in a narrow band, they may contribute to regional rainfall budget at seasonal and interannual time scales. Figure 6a presents that the corridors yield abundant precipitation in central China, with a maximum amount exceeding $350 \mathrm{~mm}$. Over there, the corridor events occurring about one-quarter of the days of the mei-yu season can produce more than one-half of the rainfall amount (Fig. 6b), suggesting a crucial contribution to rainfall budget. During the corridor events, the rainfall intensity increases by $80 \%-100 \%$ in central China, while it decreases by $20 \%$ in North China and by $60 \%$ in the South China Sea (Fig. 6c). Such a sandwich-shaped pattern with excessive rainfall in a narrow band and belownormal rainfall in nearby areas is a typical feature of corridors or mei-yu rainbands (TD06; Geng and Yamada 2007). Atmospheric circulations favorable for corridors seem to have a relatively dry condition in nearby areas (Trier et al. 2014), which is discussed later. As a comparison, the non-corridor-type events produce a rainfall amount of 350-500 mm over southeastern China (Fig. 6d). They account for $50 \%-60 \%$ of seasonal rainfall there due to an enhanced rainfall intensity by $60 \%-80 \%$ (Figs. 6e,f). However, the rainfall anomalies in the other areas are small, implying that the non-corridor-type centroids are mainly associated with local convective systems (Fig. 6f).

We further examined the interannual variations of corridors and the contribution to rainfall variance. Figure $7 \mathrm{a}$ shows that about 5 corridor events occur in one year with a standard deviation of 2.2. There are 14 days affected by corridors, which take up $23 \%$ of the total days during mei-yu season. The corridor-related rainfall $(247.2 \mathrm{~mm})$ accounts for one-half of the season rainfall amount $(483.2 \mathrm{~mm})$ (Figs. 7c,d). The numbers of corridors and their affected days exhibit evident interannual variations (Figs. 7a,b). Correspondingly, the accumulated rainfall of corridors varies largely from year to year with a standard 


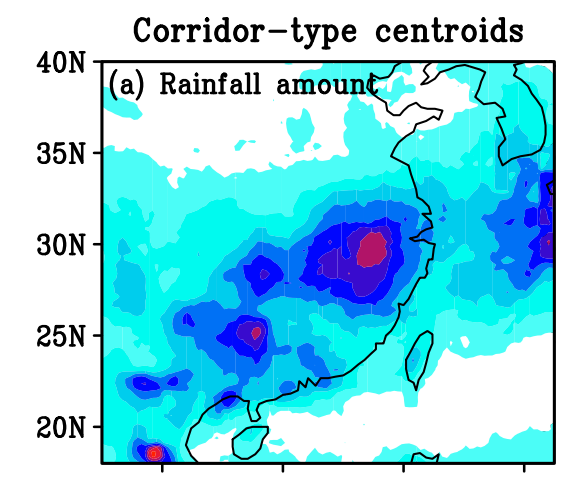

\section{Non-corridor-type centroids}
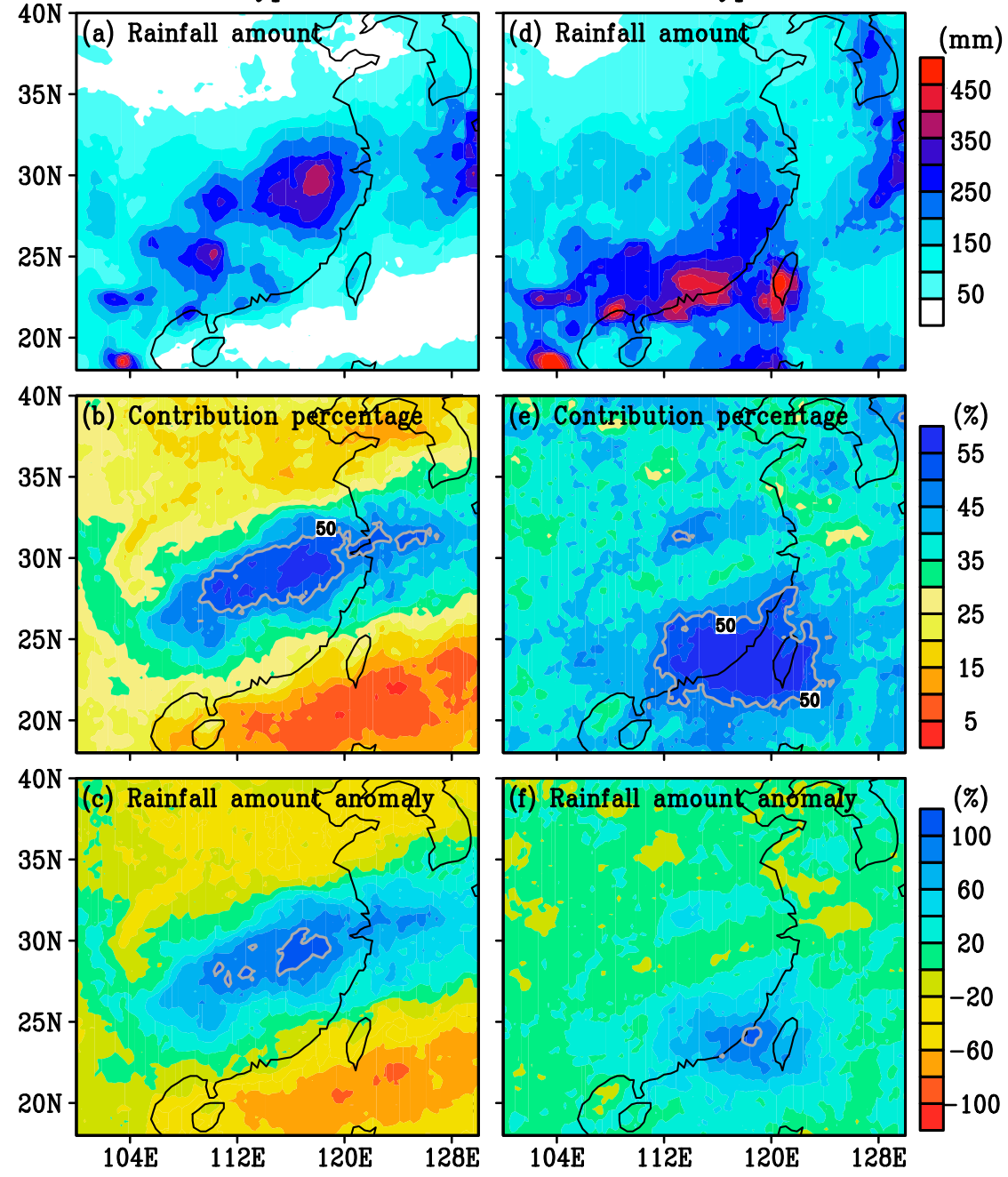

FIG. 6. (a),(d) Accumulated rainfall amount averaged over the 20 years, (b),(e) contribution ratio to seasonal rainfall (shading, with a contour of 50\%), and (c),(f) rainfall amount anomaly relative to the averaged seasonal rainfall (shading, with a contour of $100 \%$ ) by the (left) corridor-type and (right) non-corridor-type centroids.

deviation of $115 \mathrm{~mm}$ (Fig. 7c), which is comparable to that $(121.2 \mathrm{~mm})$ of the regional rainfall amount over central China (Fig. 7d). The correlation coefficient between the two series is as high as 0.84 , above the $99 \%$ confidence level (Figs. 7c,d). Overall, the rainfall by corridors (Fig. 7c) can explain $\sim 70 \%$ of the interannual variance of regional seasonal rainfall (Fig. 7d). In particular, a large rainfall amount by corridors is observed in 1998, 1999, 2010, and 2015 (Fig. 7c). The average rainfall of these four years reaches $435 \mathrm{~mm}$, nearly 2 times the mean of all corridors. They result in the anomalously wet mei-yu season with the total rainfall amount above normal (Fig. 7d). Therefore, the corridors play a dominant role in regulating both the seasonal rainfall budget and interannual variations. Their evident interannual variability may express a possible response to the anomalous large-scale circulations, which will be discussed in section 5 .

\section{Atmospheric conditions governing the diurnal cycle of rainfall corridors}

In this section, we examine the physical processes associated with the diurnal cycle of corridors. The corridors exhibit a distinct diurnal variation with either a morning peak or an afternoon feature, as revealed in section 3. To consider this discrepancy, based on the dominant peaks of the embedded centroids, we categorize the corridors into a typical group with a morning peak (65 events) and a group with a delayed afternoon peak (28 events). First, we briefly examine the rainfall patterns of the two kinds of 
(a) Corridor numbers

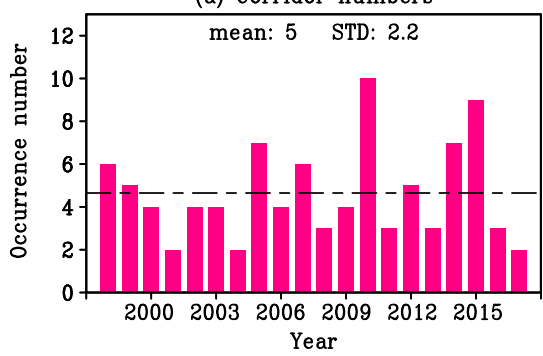

(c) Corridors' rainfall

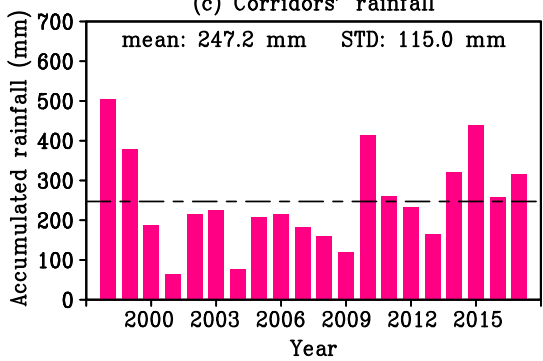

(b) Days of corridors

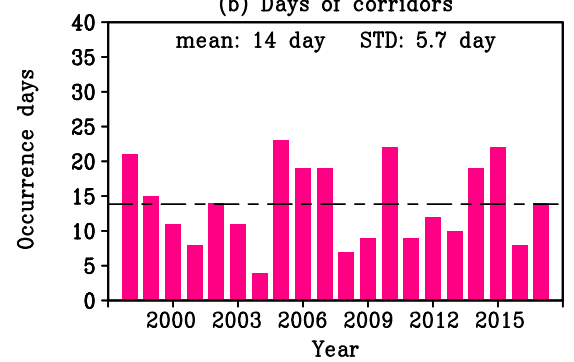

(d) Seasonal rainfall

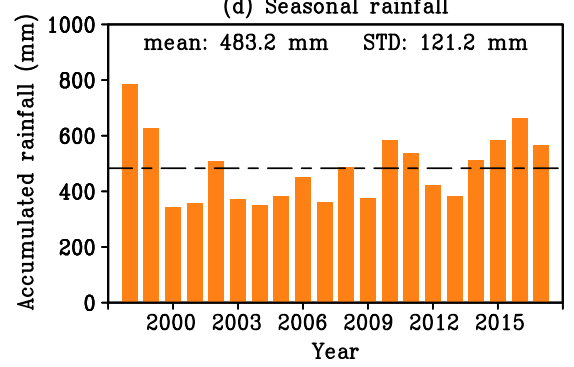

FIG. 7. Interannual variations of (a) occurrence number, (b) affected days, and (c) accumulated rainfall amount of corridors over central China. (d) Interannual variation of the rainfall amount over central China in mei-yu season. The dashed lines denote the mean value.

corridors. We then investigate the diurnally varying lowlevel winds and the mei-yu front, given their close relation to rainfall diurnal cycle.

\section{a. Rainfall patterns of the morning-peak and afternoon-peak corridors}

We examine the latitudinal distribution of rain rate from corridors that are artificially shifted to $30^{\circ} \mathrm{N}$. Figure 8 a shows that the rainfall of morning-peak corridors develops at 0200 LST and reaches the maximum during 0500-1100 LST. As for the afternoon-peak corridors, the rainfall increases later near noon and maximizes during 1400-2000 LST (Fig. 8d). Similar rainfall peaks also occur in previous days. The diurnal cycle of corridors seems to occur repeatedly under given atmospheric conditions that may persist through the corridor period.

Figures $8 \mathrm{~b}$ and $8 \mathrm{e}$ show that both types of corridors produce substantial rainfall in a west-southwest-eastnortheast-oriented band over central China. The morningpeak corridors lead to a rainfall amount that is more than 2 times that of the afternoon-peak corridors, partly due to a higher occurrence. Morning-peak corridors are also characterized by a relatively high rain rate in an extensive area (Figs. 8c,f). Nevertheless, both types of corridors exhibit sandwich-like patterns with excessive rainfall in central China but below-normal rainfall in northern and southern areas. Such a distribution indicates that the corridors may share some common large-scale features despite the discrepancy in diurnal cycle.

\section{b. Dynamic conditions associated with the two types of corridors}

In this subsection, we focus on the atmospheric conditions that regulate the diurnal variations of rainfall corridors. First, we examine the diurnally varying low-level winds considering their importance to rainfall diurnal cycle over eastern China. The composite analysis is made with respect to the latitudes of corridors, provided that both rainfall and environmental variables are artificially shifted to $30^{\circ} \mathrm{N}$. In other words, the geographic locations here are in a relatively sense but can still reflect the factual features as in TD06.

Figure 9 shows that the anomalies of low-level winds and horizontal divergence during the corridor events with the daily mean of June-July removed. A large-scale anticyclonic circulation dominates over the western Pacific. Anomalous southerly or southwesterly winds prevail over South China, the East China Sea, and southwest Japan, indicating an active monsoon flow. Anomalous northerly winds appear in the midlatitude areas (the Korean peninsula and northern China). The confluence of low-level winds forms a front or shear line stretching from central China to Japan. The enhanced low-level convergence is evident along the frontal zone, while the divergence is seen in the adjacent northern or southern areas, coinciding with the sandwich-shape rainfall pattern. Both types of rainfall corridors seem to occur with the active mei-yu front that is also recognized favorable for sustaining the East Asian summer rainband (Kodama 1993; Ding et al. 2001). 


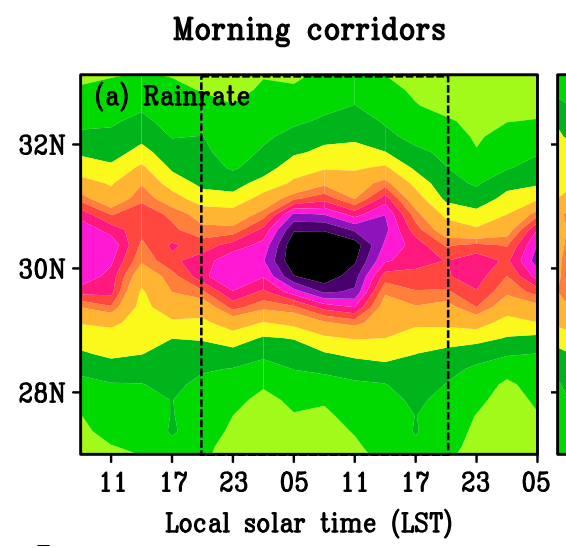

\section{Afternoon corridors}
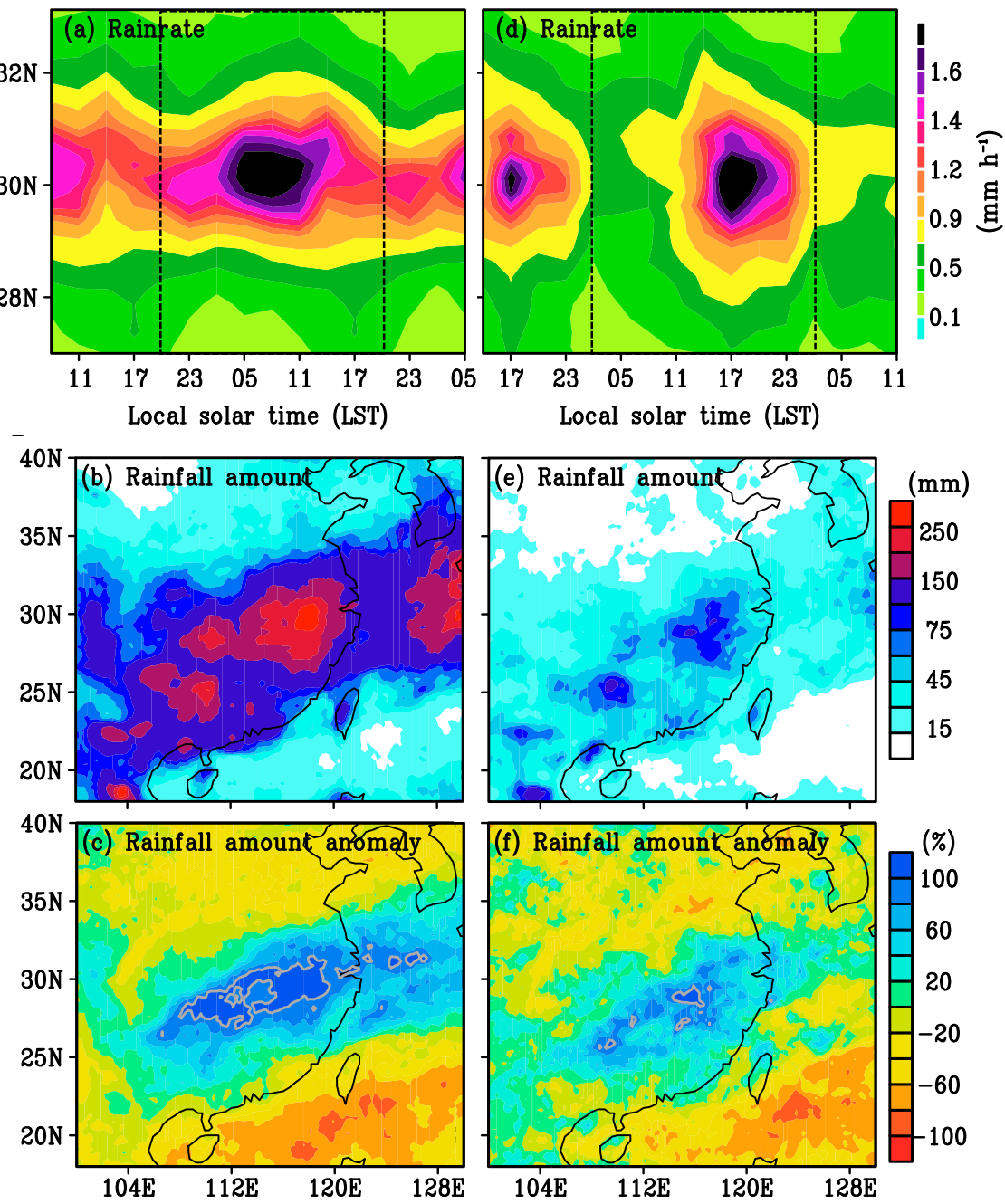

FIG. 8. (a),(d) Latitude-time diagram of rain rate (shading) averaged over $112^{\circ}-120^{\circ} \mathrm{E}$, (b),(e) accumulated rainfall amount, and (c),(f) rainfall amount anomaly percentage by (left) morning-peak and (right) afternoon-peak corridors (shading, with a contour of $100 \%$ ). All of the corridor days are composited in a 24-h window as denoted by the dashed box in (a) or (d). For a broader view, rainfall in the hours prior to/after each corridor day is shown as well.

Figure 9 also shows that the two types of corridors are characterized by the regional differences and diurnal variations in low-level winds. During the morning-peak corridors (Figs. 9a-d), the low-level winds exhibit two regional maxima: one over South China and another over the East China Sea (red contours). The western maximum of wind speed has more southerly component and accounts for a relatively strong convergence in central China, while the eastern one features more westerly component and induces convergence over Japan. At 0200 LST (Fig. 9b), the southwesterly over South China increases by $\sim 2 \mathrm{~m} \mathrm{~s}^{-1}$ and reaches $\sim 8 \mathrm{~m} \mathrm{~s}^{-1}$ (red contours). Among the morning-peak corridors, about $40 \%$ of them are characterized by the NLLJs with the maximum 925 -
$\mathrm{hPa}$ wind speed exceeding $10 \mathrm{~m} \mathrm{~s}^{-1}$ over South China at 0200 LST. Downwind of the southerly maximum (like the NLLJ's terminus), the low-level convergence is strongly strengthened in central China (shading). At 0800 LST (Fig. 9c), the southwesterly winds are still evident at South China while the northerly winds become strong in midlatitude areas, resulting in the strengthened convergence over central China. Accordingly, the rainfall grows in central China since late night and reaches its maximum at morning. The rainfall then weakens at 1400 LST along with the declined southwesterly winds in South China (Fig. 9d). The wind speed maximum over the East China Sea also experiences a nighttime acceleration, and it strengthens low-level convergence over Japan and the 
Morning corridors
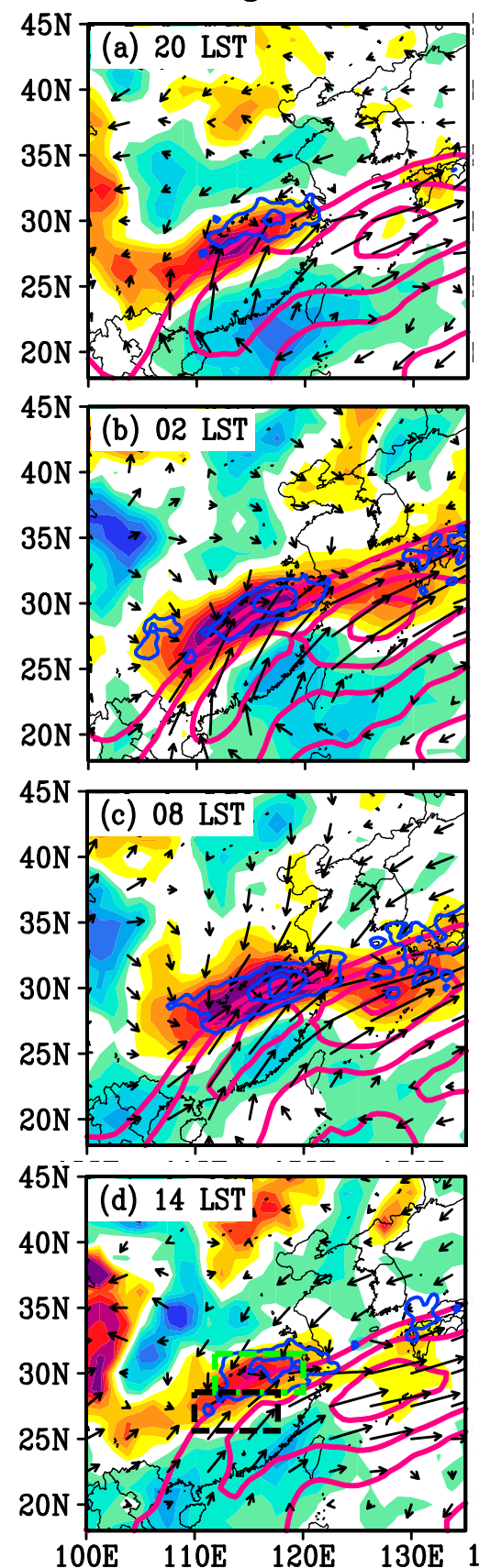

Afternoon corridors
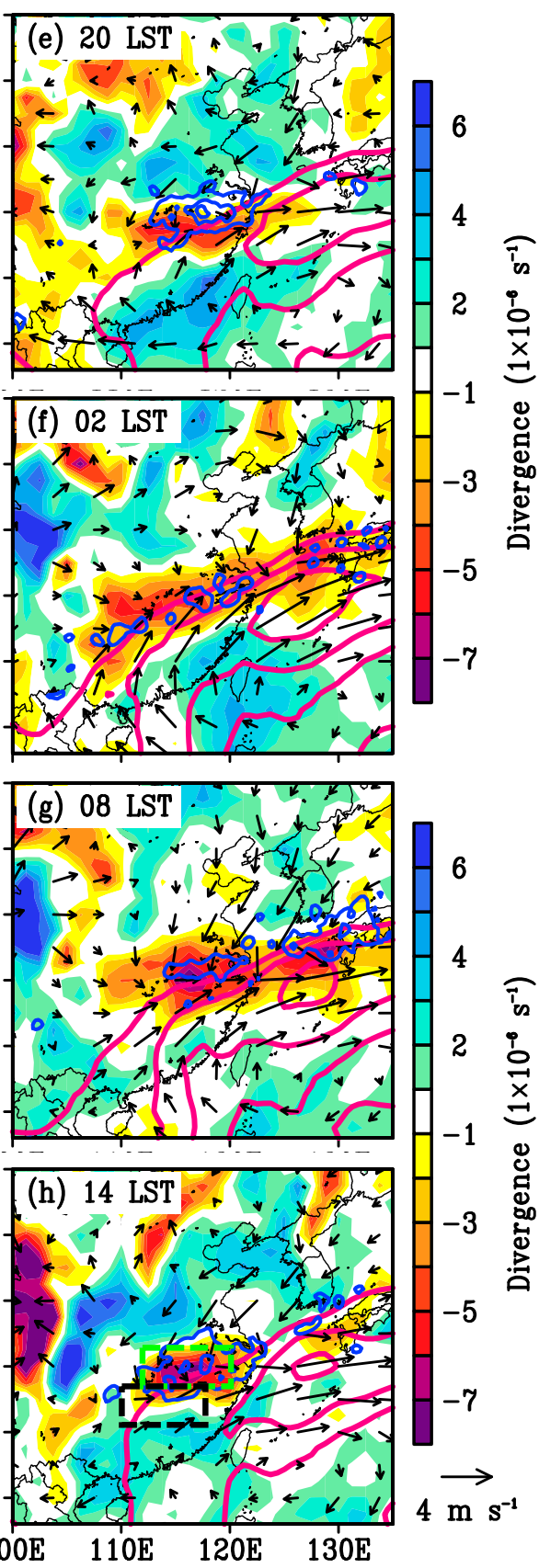

FIG. 9. (a)-(d) Composite of 925-hPa divergence anomaly (shading), horizonal wind speed (red contours of $4,6,8$, and $10 \mathrm{~m} \mathrm{~s}^{-1}$ ), horizontal winds anomaly (vectors), and rain rate (blue contours of $0.8,1.6$, and $2.4 \mathrm{~mm} \mathrm{~h}^{-1}$ ) in the morning-peak corridors. The anomalies are relative to the 20-yr climatology. (e)-(h) As in (a)-(d), but for the afternoon-peak corridors. All corridors have been artificially shifted to $30^{\circ} \mathrm{N}$. In (d) and (h), the green dashed box denotes the corridor zone $\left(112^{\circ}-120^{\circ} \mathrm{E}, 28.75^{\circ}-31.25^{\circ} \mathrm{N}\right)$ and the black dashed box denotes the southwestern area $\left(110^{\circ}-117.5^{\circ} \mathrm{E}, 26.25^{\circ}-28.75^{\circ} \mathrm{N}\right)$ used to make Fig. 10, below. 

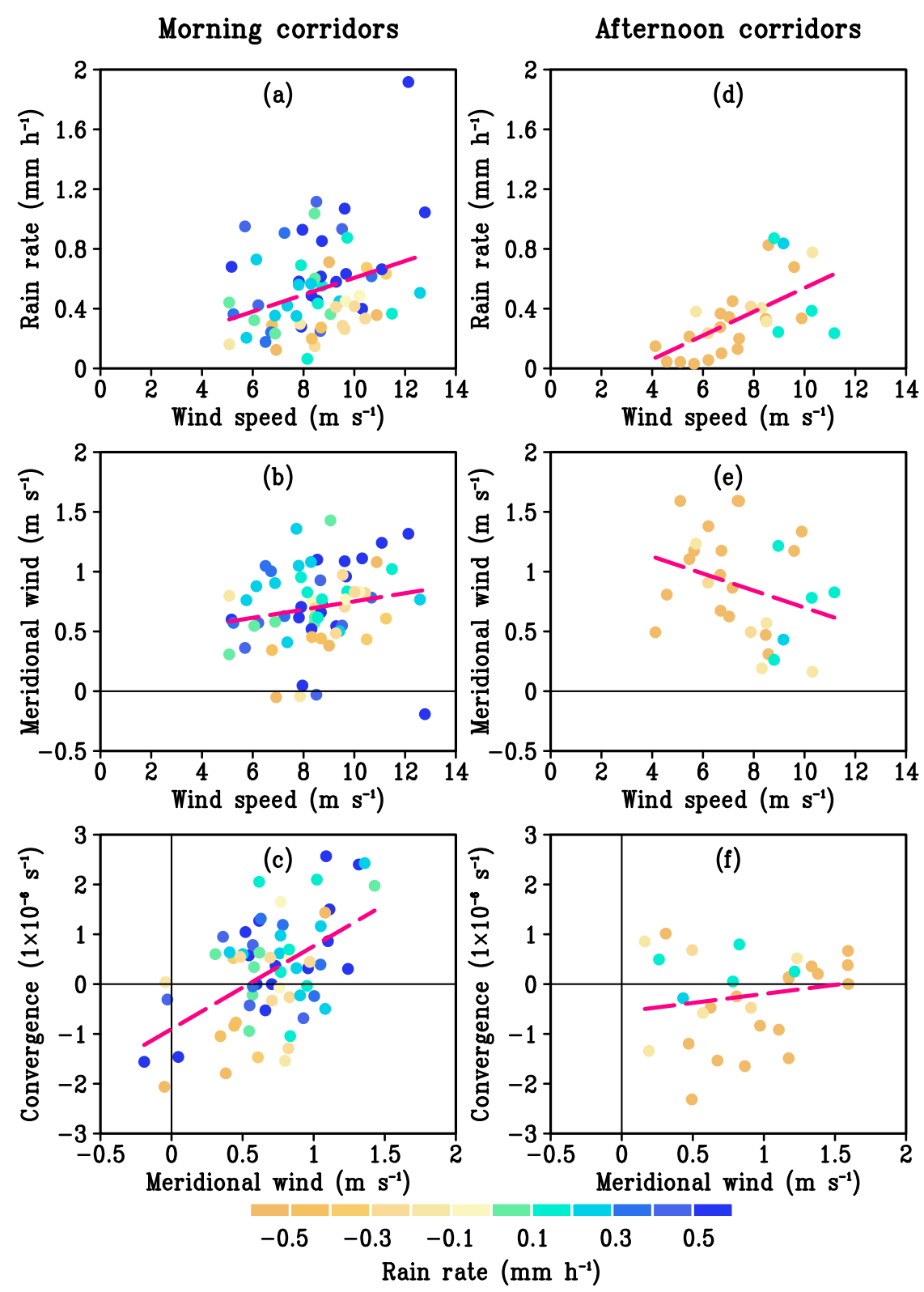

FIG. 10. Scatterplots of (a),(d) wind speed and rain rate,(b),(e) diurnal deviations of meridional wind and wind speed, and (c),(f) diurnal deviations of convergence and meridional wind averaged at $925-850 \mathrm{hPa}$ at $0200 \mathrm{LST}$ in (left) morning-peak and (right) afternoon-peak corridors. The shading denotes the rain-rate difference between morning and afternoon, and the dashed lines are the regression lines. The convergence/rain rate is averaged in the corridor zone as marked in Figs. $9 \mathrm{~d}$ and $9 \mathrm{~h}$, and the winds are averaged in the adjacent southwestern area.

southern coasts of the Korean peninsula, but at a magnitude less than that in central China (Kanada et al. 2014; Shin et al. 2019).

Figures 9e-h show that, during the afternoon-peak corridors, the regional wind maximum is still seen over East China Sea but is absent in South China. Central China is mostly located at the entrance of the wind maximum. The southerly winds strengthen at 0200 LST over
South China and the East China Sea (Fig. 9f). The nocturnal convergence is enhanced at the western coast of Japan, while it remains weak in central China (cf. Figs. 9f,e) due to the absence of the wind maximum over South China. The differences between Figs. $9 b$ and $9 f$ also highlight that the regional maximum of low-level winds (or LLJs) and its diurnal variation over South China may be the key factors controlling the diurnal cycle of 
corridors. The convergence in central China is locally enhanced later at 0800-1400 LST when the midlatitude northerlies maximize. Accordingly, the growth of rainfall systems is delayed and results in an afternoon rainfall peak.

We further examine the relationship between the lowlevel wind maxima over South China and rainfall over central China for individual corridors. Figure 10a shows that, during morning-peak corridors, the wind speed at 0200 LST is positively correlated to the rain rate, with a correlation coefficient of +0.33 . It suggests that the intensity of monsoon southwesterlies strongly affects the rainfall amount in the corridors. Meanwhile, those events with a large rainfall amount are usually associated with the relatively large rain rate in the morning hours (shading in blue). Figure 10b shows that low-level winds tend to possess a southerly deviation at 0200 LST in most of the morningpeak corridors, with a diurnal amplitude in proportion to full wind speed. Such an active monsoon flow coupling with diurnal cycle is likely due to the boundary layer inertial oscillation, which is often observed over eastern China (Chen et al. 2009, 2013; Du et al. 2014, 2015; Xue et al.2018; Fu et al. 2019; Zeng et al. 2019; Zhang et al. 2019). The strong deviation of southerly winds intensifies the lowlevel convergence in central China, especially for the corridors with substantial morning rainfall (Fig. 10c).

As for the afternoon-peak corridors, the southwesterly winds still exhibit a positive correlation with the rain rate (Fig. 10d). However, the relationship between wind speed and diurnal cycle of meridional wind is quite weak (Fig. 10e). Some weak convergence at 0200 LST is also contributed by wind deviation (Fig. 10f), but it is much weaker than that in morning-peak corridors (Fig. 10c). These results suggest that low-level winds, especially LLJs, have a significant influence on the rainfall intensity in corridors, similar to those in the United States (Augustine and Caracena 1994; TD06; Trier et al. 2006, 2014). We further emphasize that the regional wind maximum when coupling with diurnal variation in South China is important to control the rainfall diurnal cycle in corridors downstream in central China.

\section{c. Thermodynamic environment associated with the two types of corridors}

We further examine the regional features and diurnal cycles of water vapor fluxes and moist static energy. Figure 11a shows that, during the morning-peak corridors, the water vapor flux is estimated as $300-400 \mathrm{~kg} \mathrm{~m}^{-1} \mathrm{~s}^{-1}$ over South China (refer to the colors of the streamlines), but its diurnal phase is suppressed at 2000 LST (black vectors). The water vapor flux increases by $\sim 15 \%$ at 0200 LST (Fig. 11b) and coincides with the speeding up of southerly wind over South China (Fig. 9b). The moisture convergence begins to increase downstream of the wind maximum, facilitating the growth of nocturnal convection at the mei-yu frontal zone.
The moisture mainly originates in monsoon southwesterlies and partly comes from evaporation over the heated land of South China (Shinoda et al. 2005; Yamada et al. 2007a; Chen et al. 2017). At 0800 LST, both moisture convergence and precipitable water are the strongest in the corridors at central China, with a joint help of the southwesterly anomaly from South China and the northerly anomaly from midlatitudes (Fig. 11c). These conditions thus favor the maintenance of organized moist convection in the morning hours. The water vapor flux and convergence then weaken with a decayed wind speed in the afternoon, resulting in suppressed precipitation in the corridors (Fig. 11d).

Figures $12 \mathrm{a}-\mathrm{d}$ further show the vertical structure of thermodynamic variables across the morning-peak corridors. A pool of warm moist air with a high equivalent potential temperature $\theta_{e}$ is established in the lower troposphere in the southern region, and a large meridional gradient of $\theta_{e}$ (denoting the mei-yu front) is seen in the corridor latitudes. From 2000 to 0200 LST, along with the enhanced southerly wind, the high- $\theta_{e}$ airmass is accumulated in the south of the front although the $\theta_{e}$ value declines slightly due to nocturnal cooling (Fig. 12b). Around $30^{\circ} \mathrm{N}$, the highest $\theta_{e}$ is elevated at $925 \mathrm{hPa}$ and the depth of the $344-\mathrm{K}$ layer reaches $\sim 2 \mathrm{~km}(800 \mathrm{hPa})$, which is nearly 2 times that at $20^{\circ} \mathrm{N}$. The vertically tilted warm moist air is probably due to an enhanced ascending motion in the frontal zone at late night and morning (Figs. 12b,c). Such an elevated layer of warm moist air facilitates the growth of nighttime convection, which is also seen in the mesoscale modeling of corridor cases (Trier et al. 2006, 2017; Chen et al. 2017). Meanwhile, there is a large frontogenesis during 0200-0800 LST (Figs. 12b,c), which corresponds well to the confluence and deformation of low-level winds that are strengthened at night (e.g., Ninomiya 1984; Chen et al. 1994, 2009). The active mei-yu front appears to serve as an effective boundary where the monsoon southwesterlies may develop the ascending flow.

For the afternoon-peak corridors, the water vapor flux is large over the East China Sea and is relatively weak over South China (Figs. 11e-h). At 0200 LST, the flux convergence is enhanced over western Japan and is less evident over central China (Fig. 11f). The enhancement of moisture convergence and precipitable water over central China is delayed to $0800-1400$ LST (Figs. 11g,h). Figures 12e-h also show that the vertical structures are characterized by weaker diurnal variations in southerly winds, elevated high- $\theta_{e}$ air at the frontal zone, and mei-yu frontogenesis, although the daily-mean features are quite analogous to those in the morning-peak corridors. Notably, anomalously high $\theta_{e}$ of up to $354 \mathrm{~K}$ is established in the near-surface layer at the adjacent south of the corridors at 1400 LST, probably due to the thermal instability by solar heating 


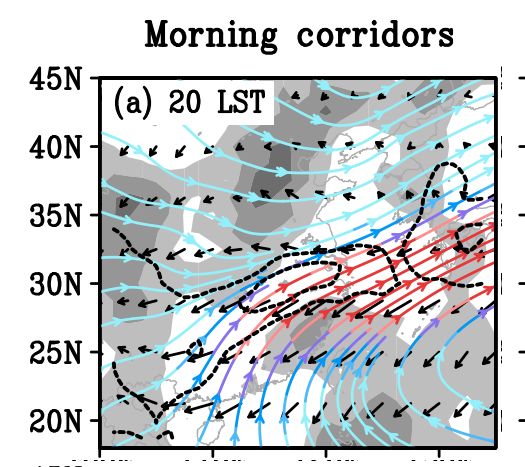

\section{Afternoon corridors}
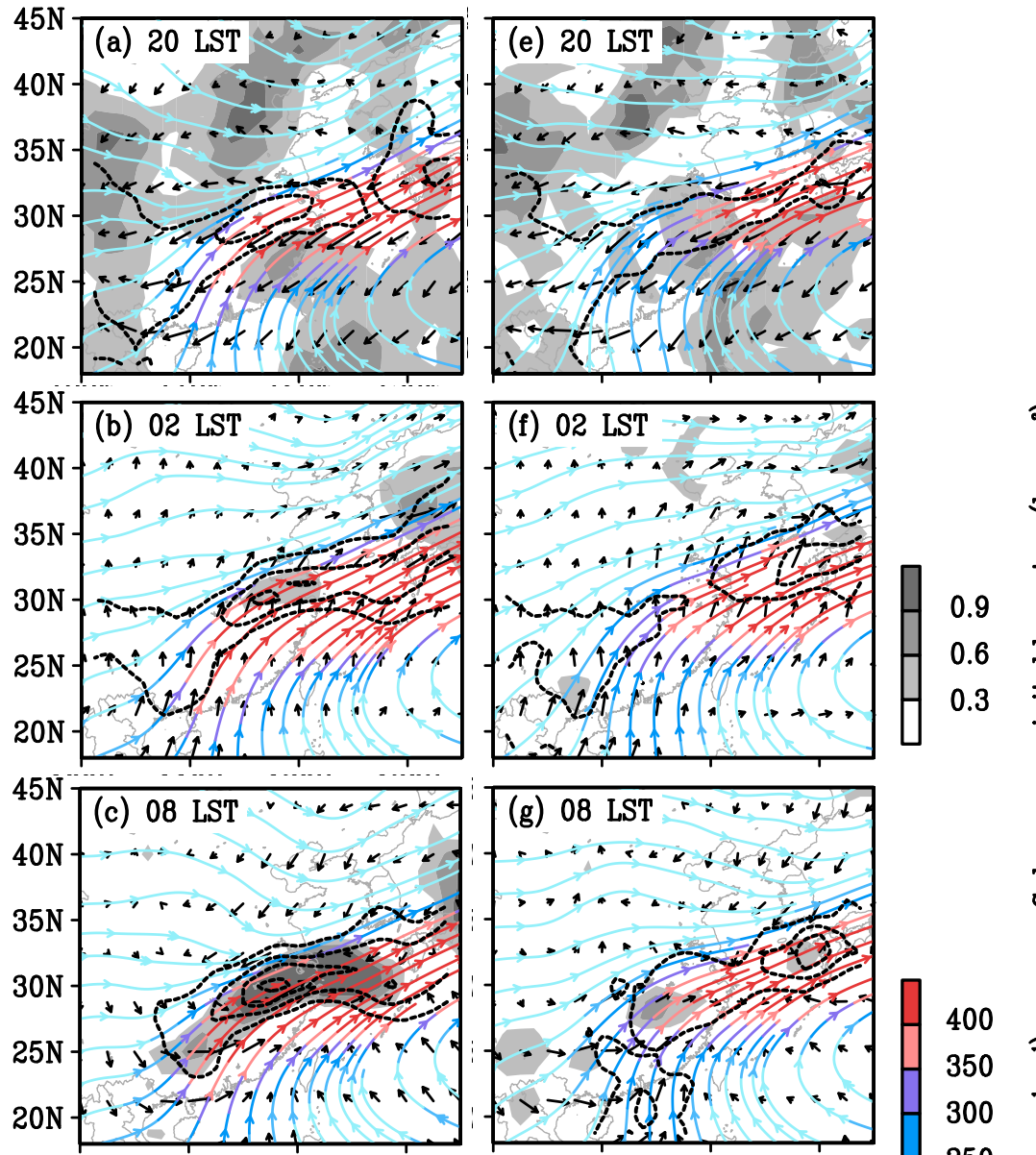

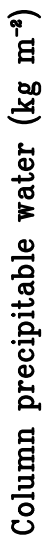
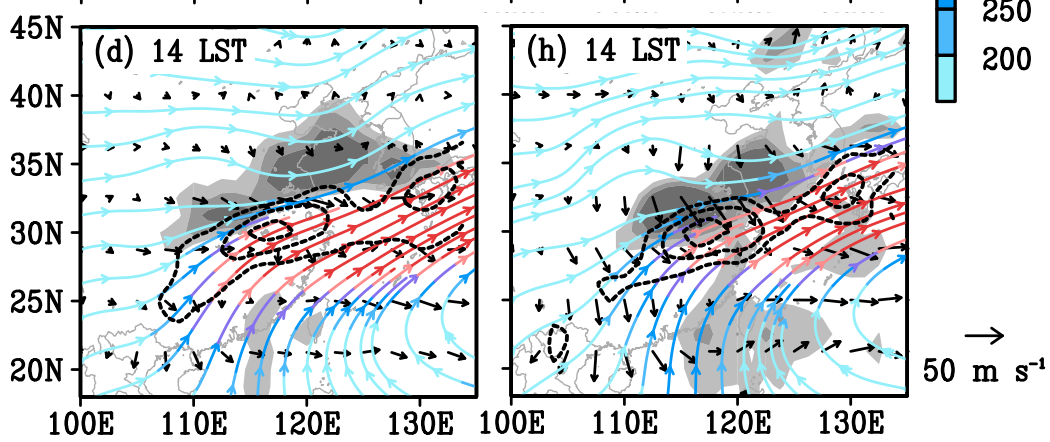

FIG. 11. Composite of the diurnal deviation of column precipitable water (shading), diurnal deviation of water vapor flux (vectors), divergence of water vapor flux (contours with interval of $8 \times 10^{-5} \mathrm{~s}^{-1}$ and start at $-32 \times 10^{-5} \mathrm{~s}^{-1}$ ), and water vapor flux (streamlines) in morningpeak corridors. (e)-(h) As in (a)-(d), but for the afternoon-peak corridors. All corridors have been artificially shifted to $30^{\circ} \mathrm{N}$.

(Fig. 12h). Therefore, in contrast to morning-peak corridors, the afternoon-peak corridors seem to be attributed to the delayed moisture convergence and partly to the daytime thermal forcings, while they are less affected by the diurnal variation of monsoon southwesterlies.

\section{Large-scale conditions associated with the persistence of rainfall corridors}

Besides the diurnal cycle due to regional forcings, another distinct feature of the rainfall corridor is the multiday development of successive MCSs. They seem 


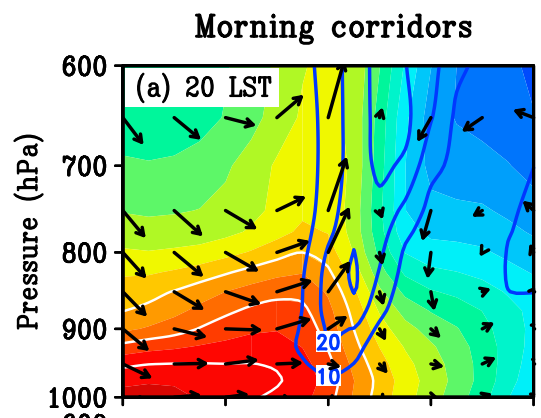

Afternoon corridors
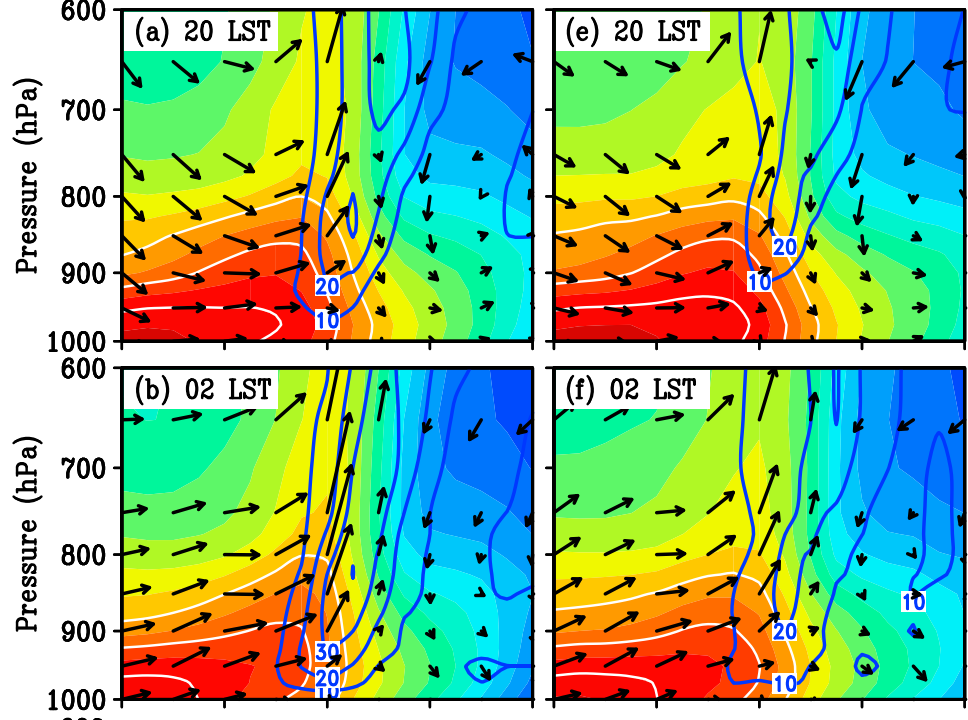

356
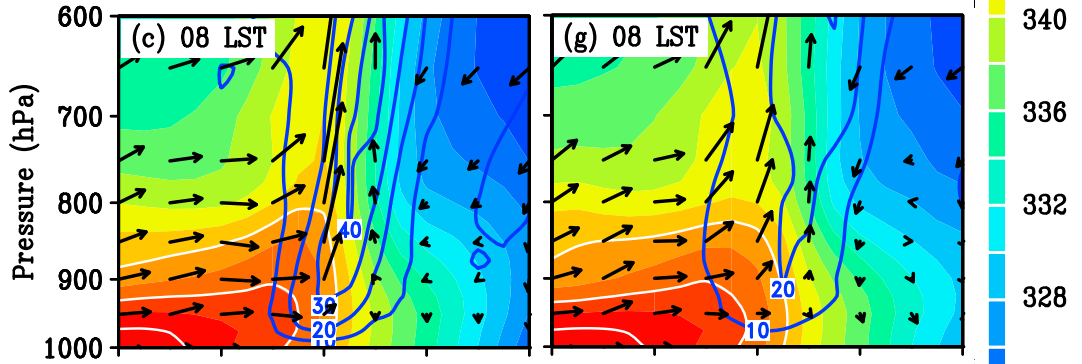

352
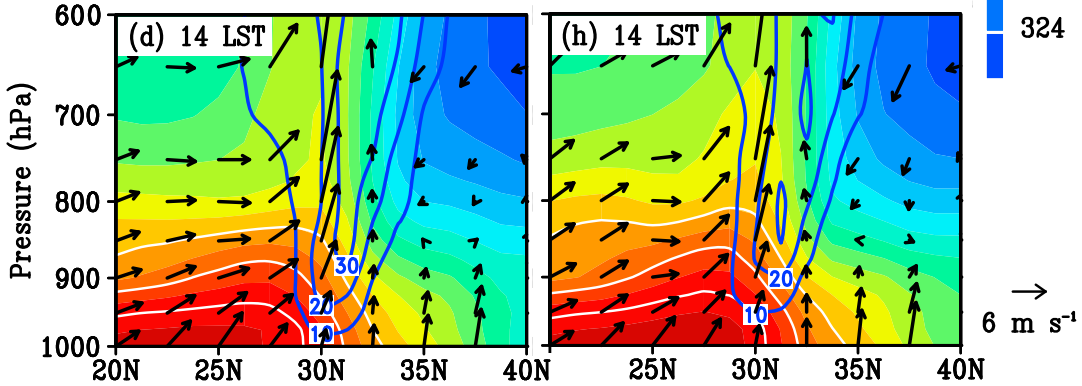

FIG. 12. Vertical section of equivalent potential temperature (shading, with white contours of 344,348 , and $352 \mathrm{~K}$ ), frontogenesis (blue contours), and in-plane flow (vectors, with vertical motion multiplied by 100 ) averaged at $112^{\circ}-120^{\circ}$ E in morning-peak corridors. (e)-(h) As in (a)(d), but for the afternoon-peak corridors. All corridors have been artificially shifted to $30^{\circ} \mathrm{N}$.

to be influenced by the large-scale forcings that may last for several days or weeks (TD06; Trier et al. 2014; Chen et al. 2017). In this section, we further investigate the mechanisms that are responsible for the maintenance of corridors. To clarify the possible causes of the variations of corridor persistence, we categorize the events into three groups with a short duration (2-3 days), medium duration ( $4-5$ days), and long duration ( $\geq 6$ days), having an occurrence of 75, 12, and 6 events, respectively. All the 93 corridors are included in the statistics because they all seem to occur under the anomalous large-scale conditions as indicated in sections 3 and 4. Again, both the rainfall and environmental variables are artificially shifted to $30^{\circ} \mathrm{N}$ in this section (except for Fig. 17, described below).

\section{a. Atmospheric conditions favorable for the various-duration corridors}

We first examine the evolution of atmospheric conditions in the lower troposphere (Fig. 13). On 1-4 days prior to the onset of corridors, an anomalous anticyclone with negative vorticity has been well established over the 
Short-duration corridors
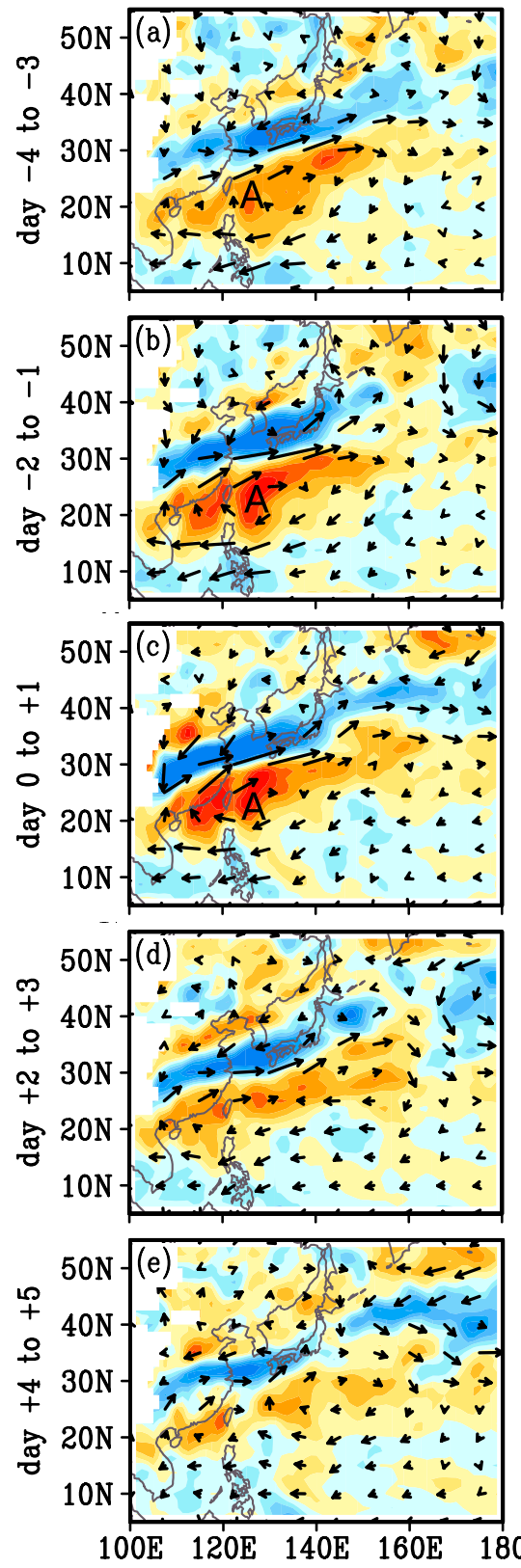

Medium-duration corridors
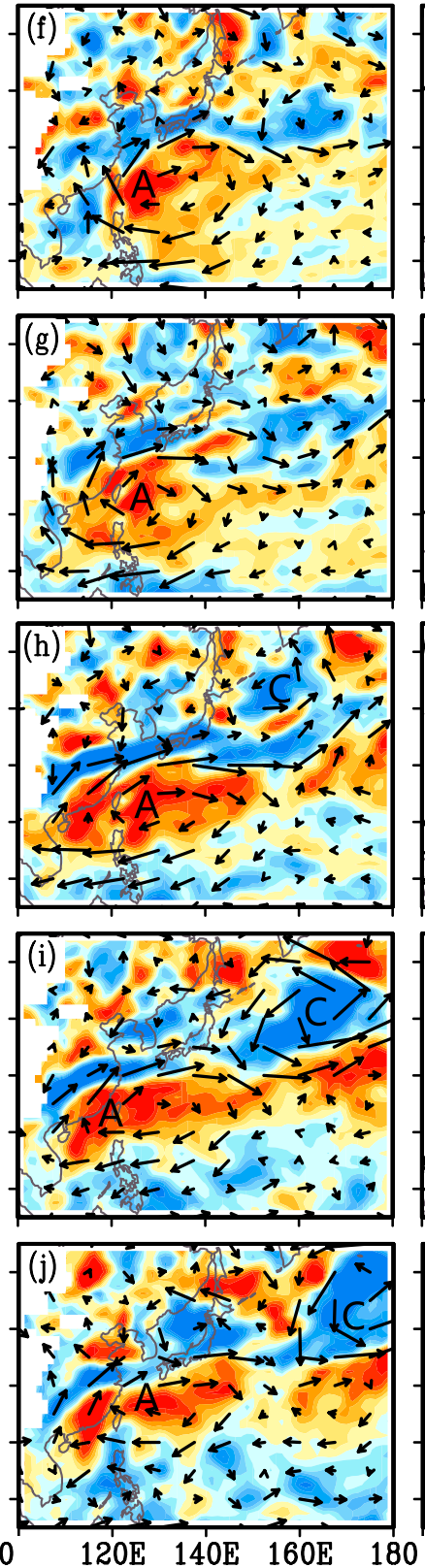

Long-duration corridors
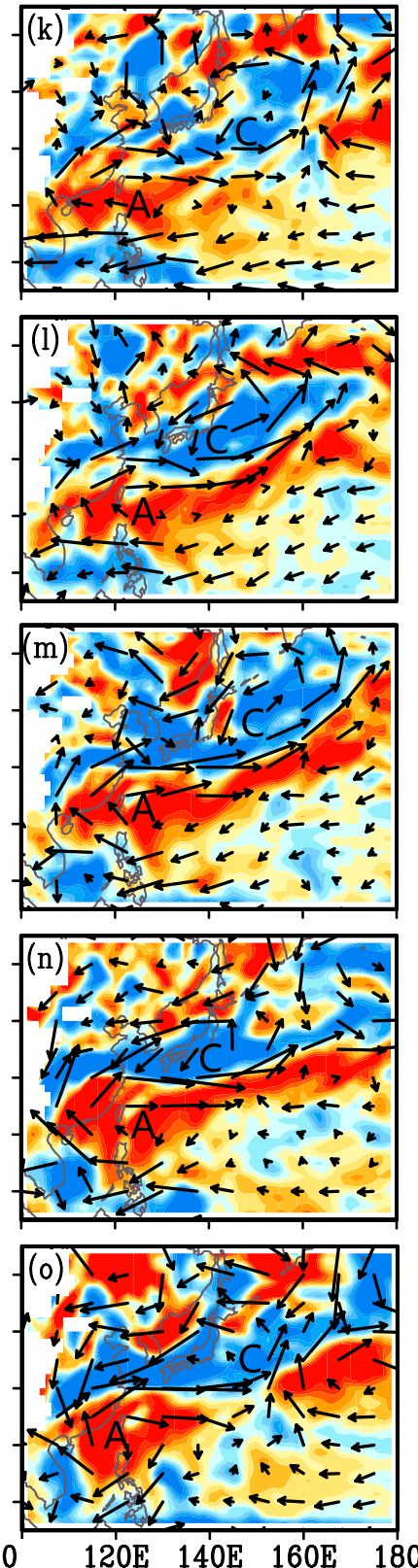

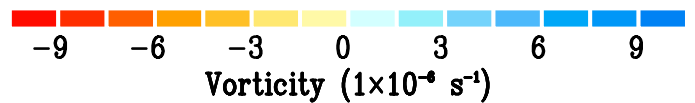

$\rightarrow$

$4 \mathrm{~m} \mathrm{~s}^{-1}$

FIG. 13. Anomalies of the 850 -hPa vorticity (shading) and horizontal winds (vectors) during (left) short-, (center) medium-, and (right) long-duration corridors. The anomalies are relative to the 20 -yr climatology. The centers of the anomalous anticyclone and cyclone are marked as "A" and "C." All corridors have been artificially shifted to $30^{\circ} \mathrm{N}$.

western Pacific (the upper two rows of Fig. 13). It gradually moves westward and dominates the northern region of the South China Sea, which is somewhat similar to the westward-propagating 20-30-day oscillation (Yang et al. 2010; R. Chen et al. 2018). On the onset day, the anomalous southwesterly winds over South China increase from $\sim 1$ to $\sim 4 \mathrm{~m} \mathrm{~s}^{-1}$ (the third row of Fig. 13). At $30^{\circ} \mathrm{N}$, a belt of anomalous cyclonic vorticity stretches eastward from central China to southern Japan and the northern Pacific. Such a dipole of an anomalous 
anticyclone/cyclone pair thus sets up a large-scale condition favorable for the enhancement of monsoon southwesterlies and the mei-yu front. It also collocates well with the spatial patterns of rainfall anomaly and lowlevel divergence/convergence (Figs. $6 c$ and 9).

A comparison of the three columns of Fig. 13 further shows that the intensity and persistence of the largescale vorticity dipole differ significantly among the three groups of corridors. During the short-duration events, the vorticity dipole decays rapidly in 2 days after the onset of corridors (Figs. 13c,d). The vorticity dipole is relatively strong during the medium-duration events and can last for up to 4 days (Figs. 13h,i). The positive vorticity at the mei-yu front then decays, as the cyclonic circulation (labeled "C") over northern Pacific moves eastward and the anticyclonic circulation (labeled "A") over western Pacific moves northward (Fig. 13j). In contrast, the dipole of anomalous cyclone and anticyclone remain nearly stationary for more than 6 days during the long-duration corridors (Figs. 13m-0). They correspond to the most sustained southwesterly winds and mei-yu front that favor the long-lived corridors. Therefore, the dipole pattern of anomalous circulations with different intensity and persistence plays a key role in regulating the duration of corridors.

We further examine the temporal variations of atmospheric variables in the middle and upper troposphere. Figure 14 shows that there is a dipole of anomalous geopotential height at $500 \mathrm{hPa}$, with a positive anomaly at $\sim 20^{\circ} \mathrm{N}$ and a negative anomaly at $\sim 40^{\circ} \mathrm{N}$. Such a meridional dipole of geopotential anomalies explains the enhanced low-level southwesterlies at low latitudes and northerly anomaly at mid- to high latitudes that are convergent to the mei-yu frontal zone. The dipole of geopotential anomalies lasts for a few days for the shortduration corridors (Fig. 14a), whereas it persists longer for the corridors with longer durations (Figs. 14b,c). In particular, the negative anomaly at $40^{\circ} \mathrm{N}$ becomes the strongest on the onset days and nearly vanishes on the ending days (blue triangle). The geopotential anomaly is instead positive at $50^{\circ}-60^{\circ} \mathrm{N}$ prior to and after the corridors, implying short-term oscillations of westerlies at mid- to high latitudes as discussed later. On the other hand, the ULJ centered at $\sim 35^{\circ} \mathrm{N}$ strengthens at the onset day of the corridors, with the maximum wind speed exceeding $\sim 35 \mathrm{~m} \mathrm{~s}^{-1}$ (purple solid). The wind speed decreases to less than $25 \mathrm{~m} \mathrm{~s}^{-1}$ on the ending day. The ULJ evolution is usually associated with the change of upper-level divergence (e.g., Wei et al. 2017). In the next subsection, we further examine the largescale atmospheric circulations associated with the ULJ and the dipole of geopotential anomalies over eastern China. (a) Short-duration corridors

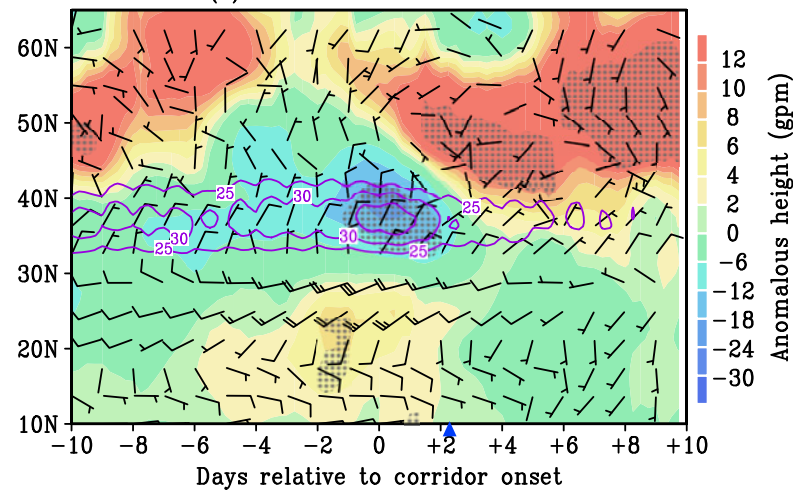

(b) Medium-duration corridors

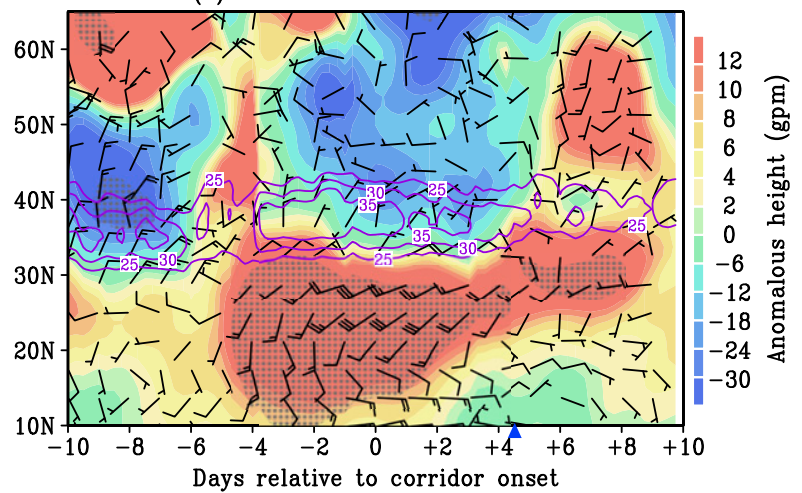

(c) Long-duration corridors

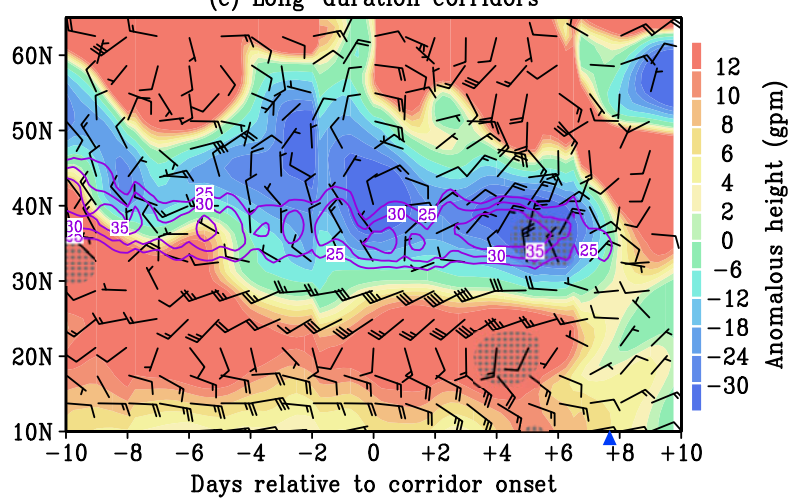

FIG. 14. Temporal variations of the 500-hPa geopotential anomaly (shading; averaged over $120^{\circ}-130^{\circ} \mathrm{E}$ ), $200-\mathrm{hPa}$ zonal wind (solid contours; averaged over $120^{\circ}-130^{\circ} \mathrm{E}$ ), and 850 -hPa horizonal wind anomaly at 0800 LST (with a long barb denoting $2 \mathrm{~m} \mathrm{~s}^{-1}$; averaged over $110^{\circ}-120^{\circ} \mathrm{E}$ ) in (a) short-duration, (b) medium-duration, and (c) long-duration corridors. The anomalies are relative to the 20 -yr climatology. The dots denote the area above the $95 \%$ confidence level. The blue triangles at the $x$ axis denote the ending days of the corridors. All corridors have been artificially shifted to $30^{\circ} \mathrm{N}$.

\section{b. Impacts of large-scale atmospheric circulation on the corridors}

Figures $15 \mathrm{a}-\mathrm{c}$ show that an anomalous ridge appears near the Ural Mountains region $\left(\sim 50^{\circ} \mathrm{E}, 40^{\circ}-60^{\circ} \mathrm{N}\right)$, while an anomalous low develops at the midlatitudes of eastern 


\section{Short-duration corridors}
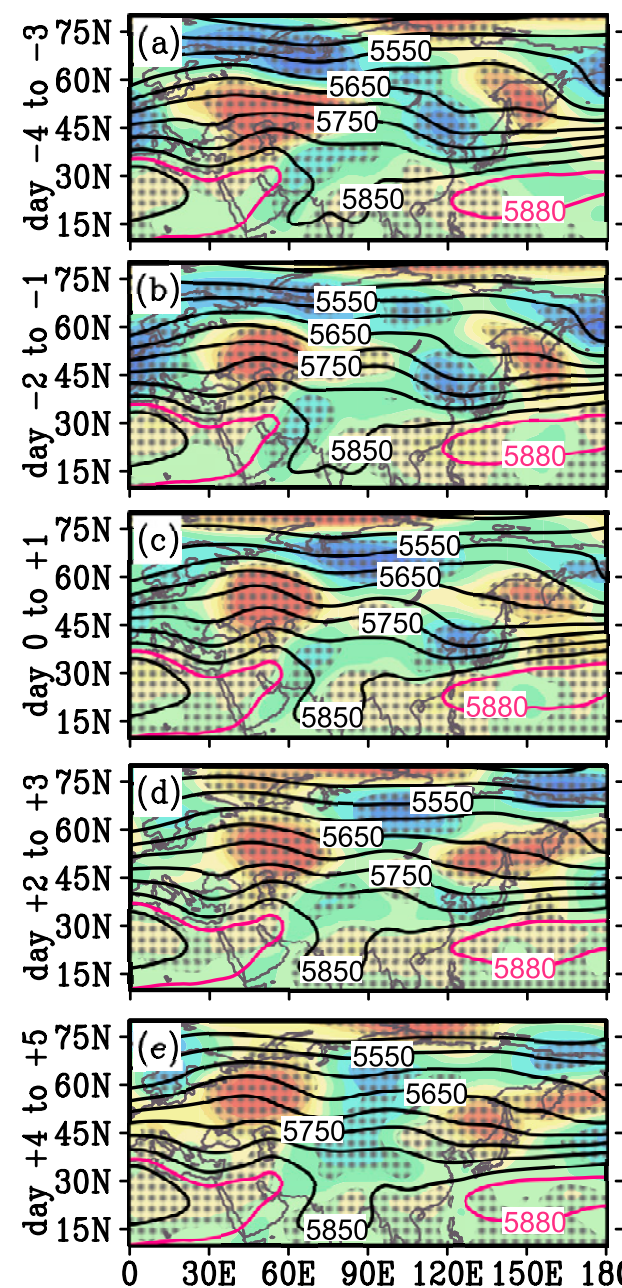

\section{Medium-duration corridors}
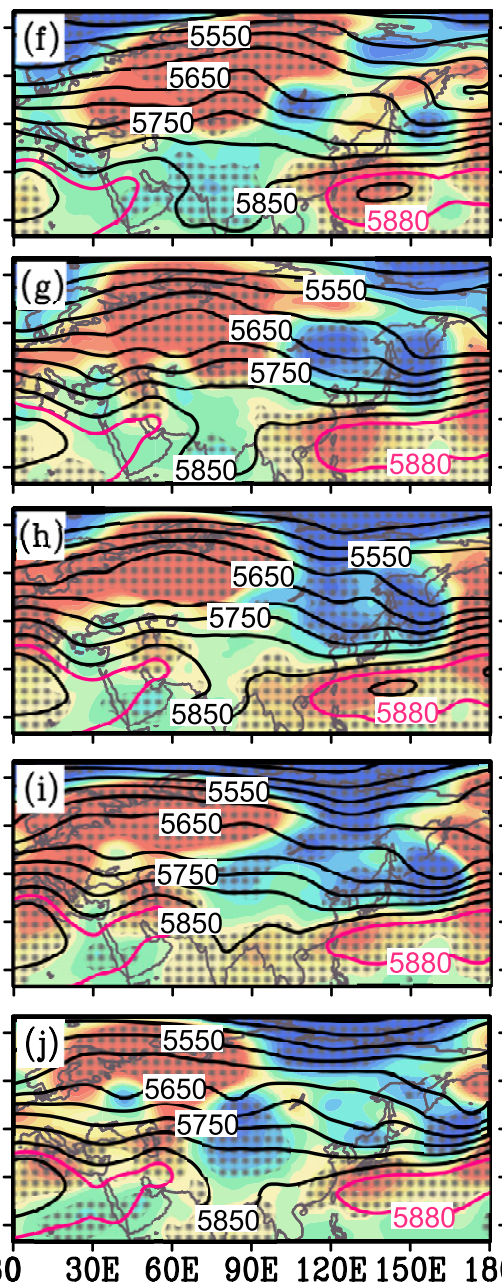

\section{Long-duration corridors}
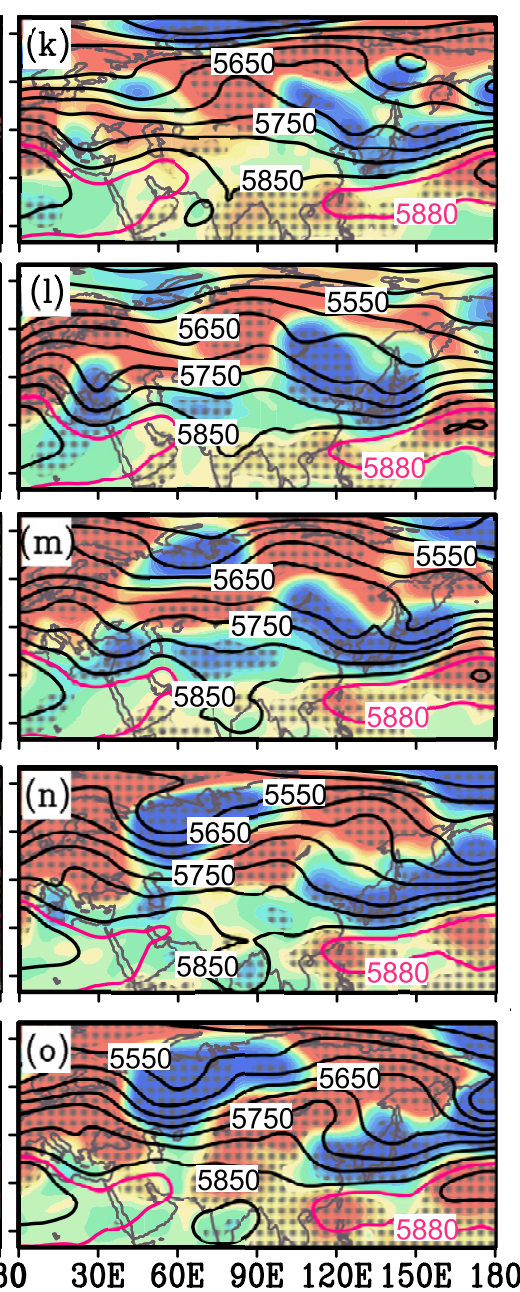

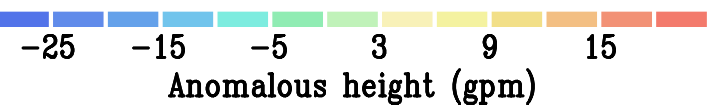

FIG. 15. Composite of 500-hPa geopotential height (contours) and its anomaly relative to the 20-yr climatology (shading) during the (left) short-, (center) medium-, and (right) long-duration corridors. The dots denote area above the $95 \%$ confidence level. All corridors have been artificially shifted to $30^{\circ} \mathrm{N}$.

China $\left(\sim 120^{\circ} \mathrm{E}, 40^{\circ}-50^{\circ} \mathrm{N}\right)$. The deepened East Asian trough along with an enhanced subtropical high over the western Pacific thus coincides with the formation of the corridors (Fig. 15c). The trough weakens in the following two days with the decay of short-duration corridors (Figs. 15d,e). A similar ridge-trough pattern of westerly fluctuations appears during the medium-duration corridors (Figs. 15f-j). The amplitudes of both ridge and trough are much larger than those during short-duration events, thereby favoring the relatively sustained corridors (Y. Chen et al. 2019). As for the long-duration corridors, Figs. 15k-o show that the anomalous ridge develops over
Europe $\left(\sim 20^{\circ} \mathrm{E}, 45^{\circ}-60^{\circ} \mathrm{N}\right)$ and another over the Siberia near Lake Baikal $\left(\sim 110^{\circ} \mathrm{E}, 45^{\circ}-60^{\circ} \mathrm{N}\right)$. The anomalous low is well established over northern China, the Korean peninsula, and Japan, where it persists for a period of up to 10 days. The Baikal ridge or blocking high is recognized to amplify the anomalous low to its east and deepen the East Asian trough, producing the mei-yu heavy rainfall (e.g., Wang 1992; Chen et al. 2007). Here, such a quasistationary low in the midlatitudes and the strengthening subtropical high in the south are shown to jointly sustain the vorticity dipole and long-lived rainfall corridors over eastern China. 


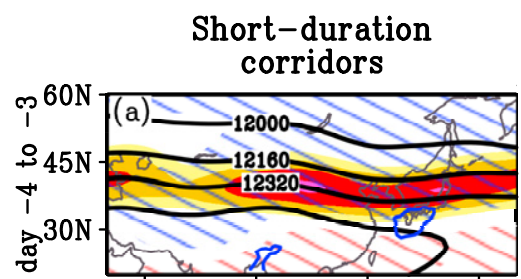

Medium-duration corridors
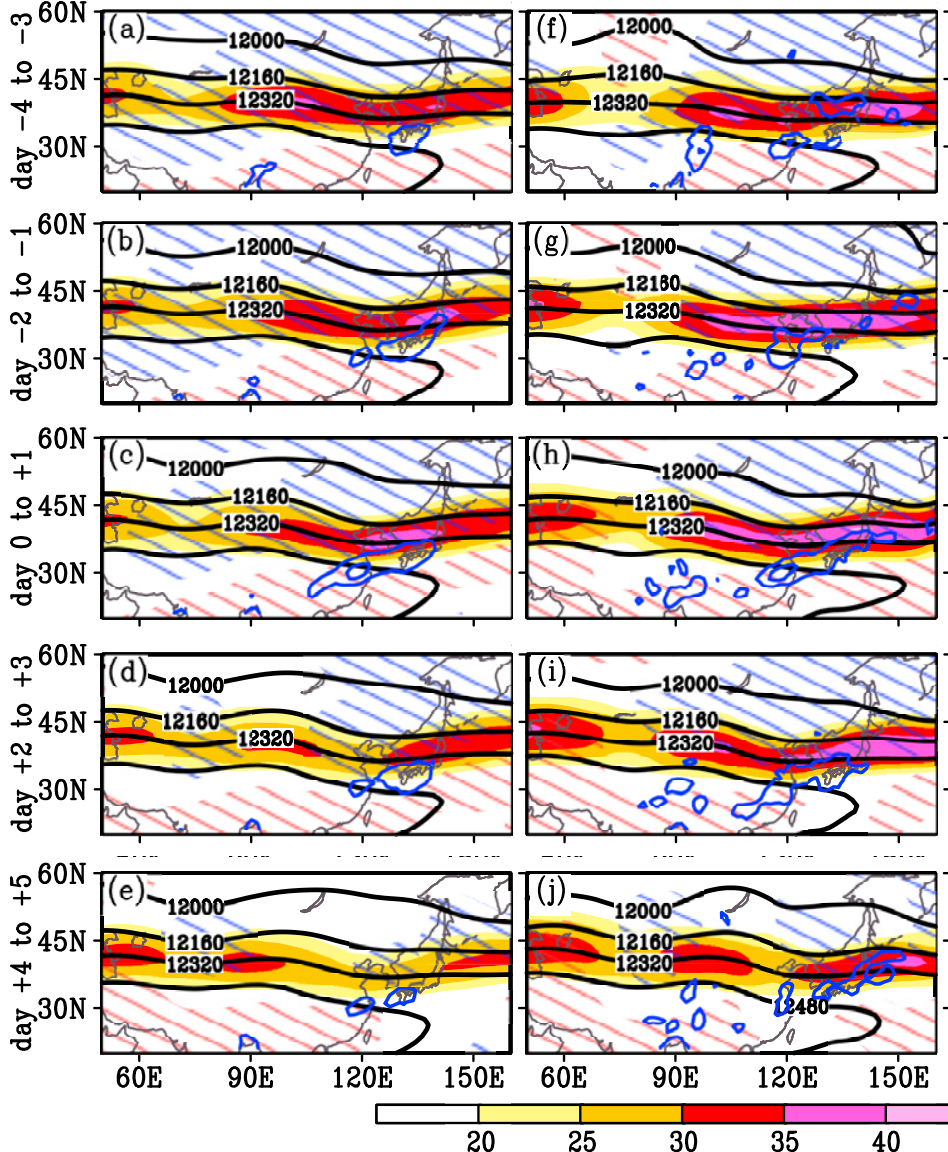
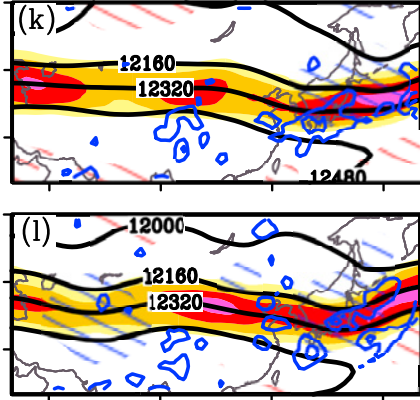

Long-duration corridors
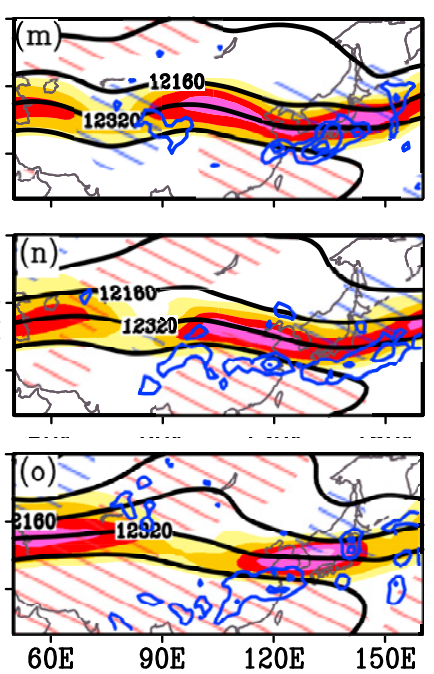

FIG. 16. Composite of 200-hPa geopotential height (black contours), divergence (blue contours of $5 \times 10^{-6}$ and $1 \times 10^{-5} \mathrm{~s}^{-1}$ ), and wind speed (shading) during the (left) short-, (center) medium-, and (right) long-duration corridors . The red or blue hatching denotes the significant positive or negative area above the $95 \%$ confidence level, respectively. All corridors have been artificially shifted to $30^{\circ} \mathrm{N}$.

Figure 16 shows the evolution of large-scale circulation in the upper troposphere. The short-duration corridors are characterized by increasing speed of ULJs over eastern China (Figs. 16a-c), in association with a strengthening SAH and an anomalous low over Siberia (hatched). The upper divergence is evidently intensified to the southern entrance of the ULJ and is favorable for convection development over central China (Fig. 16c). It is analogous to the upper-level conditions of rainfall corridors over the central U.S. plains (TD06; Trier et al. 2014). Such a collocation of upper-level divergence and low-level convergence is also evident during the active mei-yu periods (Kodama 1993; Yamazaki and Chen 1993). The ULJ and induced divergence exhibit similar patterns but at a relatively large amplitude during the medium-duration events (Figs. 16f-i). As for the longduration corridors, the ULJ has two maxima of wind speed at northern China and Japan (Figs. 16k-m). The wind speed maxima increase with the strengthened SAH (Fig. 16m). The western maximum then moves eastward and merges with the eastern one in the days after the corridor onset when the Baikal ridge develops (Figs. 16m-o). As a result, the upper-level divergence over central China is sustained for more than 6 days, producing the long-lived corridors. It is thus concluded that the dipole pattern of vorticity and geopotential anomalies in the middle to lower troposphere and the strengthened ULJ aloft play a key role in the persistence of heavy rainfall.

The large-scale atmospheric circulations governing the corridors over eastern China may be influenced by external forcings that vary from year to year. Figure 17a shows the variation of low-level vorticity dipole index, which consists of a tropical anomalous anticyclone (TAAC) and a 
(a) Vorticity dipole index

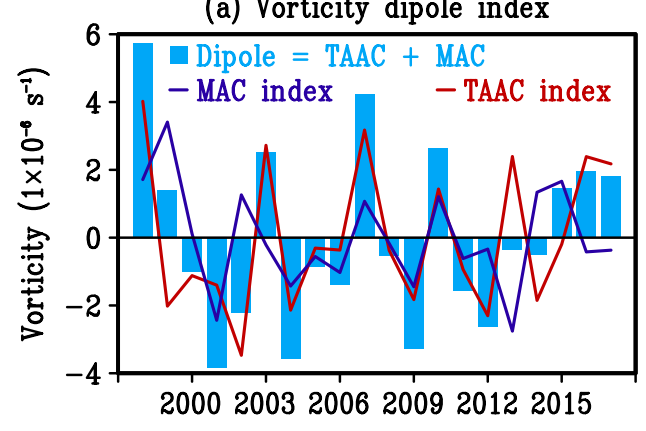

(c) Anomaly in June-July of 1998

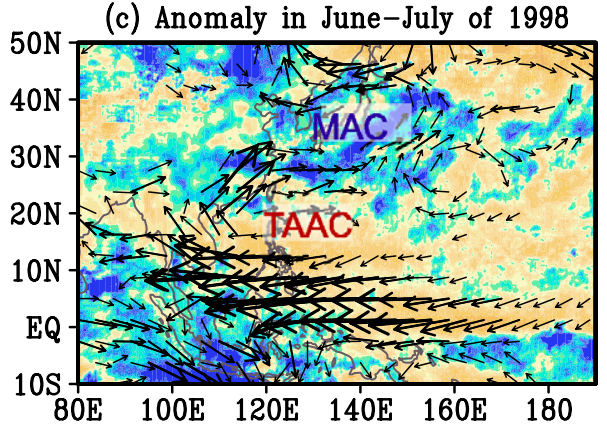

(b) Regression to TAAC index

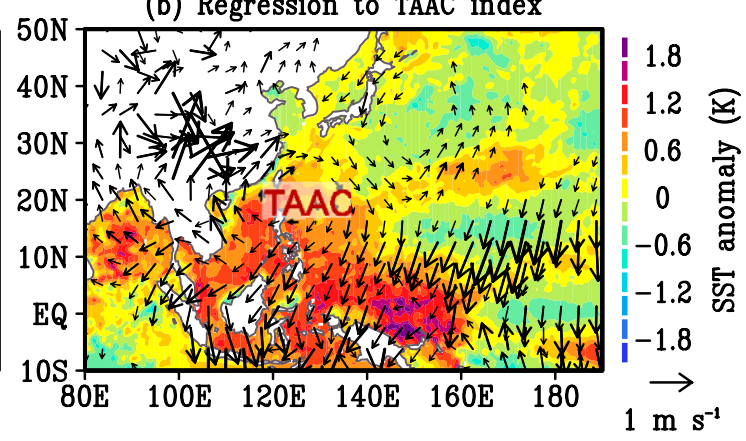

(d) Anomaly in June-July of 2016

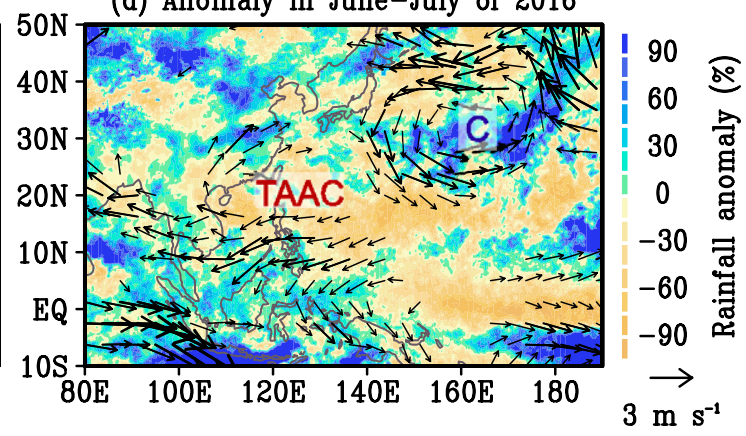

FIG. 17. (a) Interannual variations of the TAAC index (i.e., $850-\mathrm{hPa}$ vorticity anomaly averaged over $115^{\circ}-140^{\circ} \mathrm{E}$, $15^{\circ}-25^{\circ} \mathrm{N}$ and multiplied by -1 ), the MAC index (i.e., $850-\mathrm{hPa}$ vorticity anomaly averaged over $115^{\circ}-140^{\circ} \mathrm{E}$, $25^{\circ}-35^{\circ} \mathrm{N}$ ), and the dipole index (i.e., a sum of TAAC and MAC index). These key regions are chosen on the basis of Fig. 13. (b) Anomalies of sea surface temperature and $850-\mathrm{hPa}$ horizontal winds regressed to the standardized TAAC index in June-July. Also shown is the composite of rainfall anomaly and 850-hPa horizontal winds in JuneJuly of (c) 1998 and (d) 2016. The anomalies are relative to the 20-yr climatology.

midlatitude anomalous cyclone (MAC). The dipole index coincides well with the interannual variations of the day numbers (accumulated rainfall) of the corridors (cf. Fig. 17a and Figs. 7b,c), with a high correlation coefficient of 0.53 (0.71) above the $95 \%$ confidence level. The dipole index, particularly the TAAC part, is largest in the succeeding years of the El Niño events (1998, 2003, 2007, 2010, and 2016). The TAAC index is highly correlated (0.79) with the well-known Philippines anomalous anticyclone index that is defined by sea level pressure anomaly (not shown). Figure 17b shows that the TAAC activity during June and July is accompanied by an anomalous positive SST in the eastern Indian Ocean and near the Maritime Continent but an anomalous negative SST in the central-western Pacific Ocean, which usually appears during the decaying phase of El Niño events. The maintenance of the TAAC from the preceding winter to summer is probably regulated by the local air-sea interaction or the capacitor effect of the Indo-western Pacific Ocean (e.g., B. Wang et al. 2000; Xie et al. 2009, 2016).

We note that the corridors are inactive in some years (2003 and 2016) even after the El Niño events (Figs. 7b,c). In particular, the summer rainfall over central China in 2016 is less than that in 1998, although the El Niño intensity is very strong in both years (e.g., Li et al. 2017; D. Chen et al. 2019). A comparison of low-level winds shows that the TAAC is well established over the South China Sea and western Pacific in both years (Figs. 17c,d). The anomalous cyclone is dominant in midlatitudes of East Asia in 1998, but it shifts to the northern Pacific in 2016. The dipole pattern in 1998 is thus more evident than that in 2016, producing more rainfall over central China and southern Japan. Such a difference may be attributed to the circulation anomalies in midlatitudes such as the Silk Road pattern or the upper-level geopotential over the Ural Mountains region ( $\mathrm{Li}$ et al. 2017). A weak MAC index is also seen in 2003 and coincides with the relatively weak corridors (Figs. 17 and 7b,c). Therefore, the interannual variations of corridors over eastern China are strongly influenced by a joint regulation of the large-scale tropical circulations and the midlatitude westerly fluctuations. This interesting topic deserves further study to improve the prediction of severe flooding during the mei-yu season.

\section{Conclusions and discussion}

In this study, we examine a 20-yr climatology of rainfall corridors during the mei-yu season in 1998-2017. 
We also reveal the regional- and large-scale atmospheric conditions that regulate the corridors' diurnal cycle, duration, and interannual variations. The major findings are summarized as follows.

1) There are 93 corridors observed over eastern China, with a maximum occurrence in the mei-yu rainband at $27^{\circ}-31^{\circ} \mathrm{N}$. Among them, 75 corridor events last for 2-3 days, 12 events persist for 4-5 days, and 6 events last for $\geq 6$ days. These corridors yield excessive rainfall in a narrow west-southwest-east-northeast-oriented band over central China with suppressed rainfall in the adjacent south and north areas, forming a sandwichshaped pattern of rainfall anomaly. Two-thirds of the corridors exhibit a dominant peak at 0800-1100 LST, whereas the rest have a delayed peak at 1400-1700 LST. They differ from the non-corridor-type rain events that mostly maximize at 1700 LST and develop locally over South China. Although the corridors occur in less than a quarter of days in June-July, they can account for half of the seasonal rainfall amount over central China. The corridor-induced rainfall also varies largely from year to year, which explains $\sim 70 \%$ of the interannual variance of mei-yu-season rainfall. Therefore, the rainfall corridors have significant impacts on regional hydrological cycle.

2) To clarify the processes that govern the diurnal cycle, we compare the composite of atmospheric conditions between morning- and afternoon-peak corridors. The morning-peak corridors feature low-level southwesterlies with a regional maximum over South China that strengthen at late night and transport moisture to central China. Meanwhile, the mei-yu front intensifies after late night and acts as an effective boundary by which enhanced moist southwesterlies converge to promote nocturnal convection. The diurnal amplitude of southwesterlies is correlated with the background wind speed, suggesting that monsoon flow coupled with regional forcings plays a role in the formation of morning-peak corridors. In contrast, during the afternoon-peak corridors, the regional wind maximum is less evident over South China and its coupling with the rainfall diurnal variation is much weaker. The nighttime enhancement of the mei-yu front and the elevated moist airmass in frontal zone becomes relatively weak. As a result, the growth of moist convection is delayed to late morning of the next day and produces a rainfall maximum in early afternoon, which is partly supported by enhanced daytime solar heating.

3) To reveal the atmospheric circulations that regulate the duration of the corridors, we make composite analysis of the short-, medium-, and long-duration corridors. The variations in the corridors' durations are shown to de- pend on the persistence of the southwesterly winds and the mei-yu front. The atmospheric circulation features a meridional dipole with an anomalous anticyclone over the tropical western Pacific and an anomalous cyclone in the midlatitudes over East Asia. The sustaining days of the dipole coincide with the durations of corridors. The anomalous cyclone is usually accompanied by an anomalous ridge near the Ural Mountain region during short-duration corridors. Similar patterns but at a larger amplitude are seen during medianduration corridors. During long-duration corridors, an anomalous ridge is established near Lake Baikal, which helps to deepen the East Asian trough and to sustain a long-lived anomalous cyclone of the dipole. The duration of the corridors is also affected by the strengthening ULJ with the upper divergence over central China. The tropical component of the dipole is found to relate to the well-known Philippines anomalous anticyclone that may last until the summer after the El Niño events. Rainfall corridors are active (inactive) in the El Niño-decaying summers of 1998 and 2010 (2003 and 2016) when the tropical anomalous anticyclone is coupled (decoupled) with the midlatitude anomalous cyclone. Therefore, the accumulated days and rainfall of corridors are highly correlated with the interannual variation of dipole intensity.

Like the U.S. rainfall corridors, the East Asian corridors are interesting from a climate standpoint because of both the sizeable fraction of regional warm-season precipitation they account for and the negative precipitation anomalies often experienced in adjacent areas (TD06; Trier et al. 2014). This study notes that corridors exhibit some similarities and discrepancies between the two regions. Most of the corridors prefer to occur in the northern terminus of the NLLJ, and they are subject to the influence of large-scale environmental factors. One of the differences is that the corridors have two diurnal peaks over central China, while they possess a single nocturnal peak over the central United States as a result of the rainfall systems propagating from the Rockies (Carbone et al. 2002). Although the rainfall systems also propagate eastward over central China, another mode of rainfall can develop locally at night as a result of monsoon flow (Chen et al. 2012, 2017). Another difference is that the corridors over China occur at lower latitudes $\left(\sim 30^{\circ} \mathrm{N}\right)$ than those in the United States $\left(\sim 38^{\circ} \mathrm{N}\right)$. This may relate to the southward-located mei-yu front and LLJ under the East Asian trough. The corridors over East Asia are also regulated by the El Niño or midlatitude fluctuations that influence the meridional dipole. Further studies are needed to clarify the roles of topography and large-scale circulations in the corridors over the two regions. 
We also note that the NLLJ forms in a large-scale monsoon flow overpassing the heated landmass of South China to trigger nocturnal MCSs over central China (Yamada et al. 2007a,b; Chen et al. 2017). The regional forcings may couple with large-scale conditions to play a role in the development of corridor-type convection. The long-term variabilities of the summer monsoon or LLJ may lead to some trends in the observed MCSs (Feng et al. 2016). In this study, corridors are also shown to relate to the atmospheric conditions in both tropics and mid- to high latitudes, suggesting that the connection across latitudes may play a role. On the other hand, some case studies show that the cold pool or mesoscale vorticity induced by organized convection may in turn enhance the surface front. Such a local feedback may facilitate the growth of subsequent MCSs, producing persistent heavy rainfall (Luo et al. 2014; Xue et al. 2018; Zhang et al. 2018). Further studies on the multiscale processes during corridor events are warranted to improve our understanding and prediction of regional climate and extreme weather (Trier et al. 2014; Wang et al. 2014).

Acknowledgments. The authors thank Dr. Kun Zhao of Nanjing University and Dr. Lanqiang Bai of Sun Yat-sen University for informal reviews. They are grateful to NASA Goddard Space Flight Center for providing TRMM rainfall data and to the Japan Meteorological Agency for providing reanalysis data. This study was supported by the National Key Research and Development Program of China (Grant 2016YFA0600704) and the National Natural Science Foundation of China (Grants 41575068 and 41530530).

\section{REFERENCES}

Augustine, J., and K. Howard, 1991: Mesoscale convective complexes over the United States during 1986 and 1987. Mon. Wea. Rev., 119, 1575-1589, https://doi.org/10.1175/1520-0493(1991) 119<1575:MCCOTU > 2.0.CO;2.

— turnal MCS development over the central United States. Wea. Forecasting, 9, 116-135, https://doi.org/10.1175/1520-0434(1994) 009<0116:LTPTNM > 2.0.CO;2.

Bao, X., and F. Zhang, 2013: Impacts of the mountain-plains solenoid and cold pool dynamics on the diurnal variation of warmseason precipitation over northern China. Atmos. Chem. Phys., 13, 6965-6982, https://doi.org/10.5194/acp-13-6965-2013.

Carbone, R., and J. Tuttle, 2008: Rainfall occurrence in the U.S. warm season: The diurnal cycle. J. Climate, 21, 4132-4146, https://doi.org/10.1175/2008JCLI2275.1.

,,-- D. Ahijevych, and S. Trier, 2002: Inferences of predictability associated with warm season precipitation episodes. J. Atmos. Sci., 59, 2033-2056, https://doi.org/10.1175/15200469(2002)059<2033:IOPAWW>2.0.CO;2.

Chen, D., Y. Gao, and H. Wang, 2019: Why was the August rainfall pattern in the East Asia-Pacific Ocean region in 2016 different from that in 1998 under a similar preceding El Niño background? J. Climate, 32, 5785-5797, https://doi.org/10.1175/JCLI-D-18-0589.1.

Chen, G., W. Sha, and T. Iwasaki, 2009: Diurnal variation of precipitation over southeastern China: 2. Impact of the diurnal monsoon variability. J. Geophys. Res., 114, D21105, https:// doi.org/10.1029/2009JD012181.

$\_,-, \ldots$, and K. Ueno, 2012: Diurnal variation of rainfall in the Yangtze River valley during the spring-summer transition from TRMM measurements. J. Geophys. Res., 117, D06106, https://doi.org/10.1029/2011JD017056.

,,-- M. Sawada, and T. Iwasaki, 2013: Influence of summer monsoon diurnal cycle on moisture transport and precipitation over eastern China. J. Geophys. Res. Atmos., 118, 3163 3177, https://doi.org/10.1002/JGRD.50337.

—, T. Iwasaki, H. Qin, and W. Sha, 2014: Evaluation of the warm-season diurnal variability over East Asia in recent reanalyses JRA-55, ERA-Interim, NCEP CFSR, and NASA MERRA. J. Climate, 27, 5517-5537, https://doi.org/10.1175/ JCLI-D-14-00005.1.

_ W. Sha, T. Iwasaki, and Z. Wen, 2017: Diurnal cycle of a heavy rainfall corridor over East Asia. Mon. Wea. Rev., 145, 3365-3389, https://doi.org/10.1175/MWR-D-16-0423.1.

—, R. Lan, W. Zeng, H. Pan, and W. Li, 2018: Diurnal variations of rainfall in surface and satellite observations at the monsoon coast (South China). J. Climate, 31, 1703-1724, https://doi.org/ 10.1175/JCLI-D-17-0373.1.

Chen, G. T.-J., C.-C. Wang, and A.-H. Wang, 2007: A case study of subtropical frontogenesis during a blocking event. Mon. Wea. Rev., 135, 2588-2609, https://doi.org/10.1175/MWR3412.1.

Chen, H., R. Yu, J. Li, W. Yuan, and T. Zhou, 2010: Why nocturnal long-duration rainfall presents an eastward-delayed diurnal phase of rainfall down the Yangtze River valley. J. Climate, 23, 905-917, https://doi.org/10.1175/2009JCLI3187.1.

,-- , and Y. Shen, 2016: A new method to compare hourly rainfall between station observations and satellite products over central-eastern China. J. Meteor. Res., 30, 737-757, https:// doi.org/10.1007/s13351-016-6002-5.

Chen, R., Z. Wen, and R. Lu, 2018: Large-scale circulation anomalies and intraseasonal oscillations associated with longlived extreme heat events in South China. J. Climate, 31, 213 232, https://doi.org/10.1175/JCLI-D-17-0232.1.

Chen, S.-J., W. Wang, K.-H. Lau, Q.-H. Zhang, and Y.-S. Chung, 2000: Mesoscale convective systems along the meiyu front in a numerical model. Meteor. Atmos. Phys., 75, 149-160, https:// doi.org/10.1007/s007030070002.

Chen, Y., and P. Zhai, 2013: Persistent extreme precipitation events in China during 1951-2010. Climate Res., 57, 143-155, https://doi.org/10.3354/cr01171.

$\longrightarrow$, and — 2014: Two types of typical circulation pattern for persistent extreme precipitation in central-eastern China. Quart. J. Roy. Meteor. Soc., 140,1467-1478, https://doi.org/10.1002/qj.2231.

,-- , L. Zhen, and L. Li, 2019: Persistent precipitation extremes in the Yangtze River Valley prolonged by opportune configuration among atmospheric teleconnections. Quart. J. Roy. Meteor. Soc., 145, 2603-2626, https://doi.org/10.1002/ qj.3581.

Chen, Y.-L., X. Chen, and Y.-X. Zhang, 1994: A diagnostic study of the low-level jet during TAMEX IOP 5. Mon. Wea. Rev., 122, 2257-2284, https://doi.org/10.1175/1520-0493(1994)122<2257: ADSOTL $>2.0 . \mathrm{CO} ; 2$.

Ding, Y., and J. C. L. Chan, 2005: The East Asian summer monsoon: An overview. Meteor. Atmos. Phys., 89, 117-142, https:// doi.org/10.1007/s00703-005-0125-z. 
_ , Y. Zhang, Q. Ma, and G. Hu, 2001: Analysis of the large-scale circulation features and synoptic systems in East Asia during the intensive observation period of GAME/HUBEX. J. Meteor. Soc. Japan, 79, 277-300, https://doi.org/10.2151/jmsj.79.277.

Du, Y., Q. Zhang, Y. Chen, Y. Zhao, and X. Wang, 2014: Numerical simulations of spatial distributions and diurnal variations of lowlevel jets in China during early summer. J. Climate, 27, 57475767, https://doi.org/10.1175/JCLI-D-13-00571.1.

—, R. Rotunno, and Q. Zhang, 2015: Analysis of WRFsimulated diurnal boundary layer winds in eastern China using a simple 1D model. J. Atmos. Sci., 72, 714-727, https:// doi.org/10.1175/JAS-D-14-0186.1.

Feng, Z., L. Leung, S. Hagos, R. Houze, C. Burleyson, and K. Balaguru, 2016: More frequent intense and long-lived storms dominate the springtime trend in central U.S. rainfall. Nat. Commun., 7, 13429, https://doi.org/10.1038/ncomms13429.

_ , R. Houze, L. Leung, F. Song, J. Hardin, J. Wang, W. Gustafson, and C. Homeyer, 2019: Spatiotemporal characteristics and largescale environments of mesoscale convective systems east of the Rocky Mountains. J. Climate, 32, 7303-7328, https://doi.org/ 10.1175/JCLI-D-19-0137.1.

Fritsch, J., R. Kane, and C. Chelius, 1986: The contribution of mesoscale convective weather systems to the warm-season precipitation in the United States. J. Climate Appl. Meteor., 25, 1333-1345, https://doi.org/10.1175/1520-0450(1986)025<1333: TCOMCW $>2.0 . \mathrm{CO} ; 2$.

Fu, P., K. Zhu, K. Zhao, B. Zhou, and M. Xue, 2019: Role of the nocturnal low-level jet in the formation of the morning precipitation peak over the Dabie Mountains. Adv. Atmos. Sci., 36, 15-28, https://doi.org/10.1007/s00376-018-8095-5.

Geng, B., and H. Yamada, 2007: Diurnal variations of the meiyu/baiu rain belt. SOLA, 3, 61-64, https://doi.org/10.2151/sola.2007-016.

Groisman, P., R. Knight, and T. Karl, 2012: Changes in intense precipitation over the central United States. J. Hydrometeor. 13, 47-66, https://doi.org/10.1175/JHM-D-11-039.1.

Huffman, G., and Coauthors, 2007: The TRMM Multisatellite Precipitation Analysis (TMPA): Quasi-global, multiyear, combined-sensor precipitation estimates at fine scales. J. Hydrometeor., 8, 38-55, https://doi.org/10.1175/JHM560.1.

Jiang, X., N.-C. Lau, and S. Klein, 2006: Role of eastward propagating convection systems in the diurnal cycle and seasonal mean of summertime rainfall over the U.S. Great Plains. Geophys. Res. Lett., 33, L19809, https://doi.org/10.1029/2006GL027022.

Joyce, R., J. Janowiak, P. Arkin, and P. Xie, 2004: CMORPH: A method that produces global precipitation estimates from passive microwave and infrared data at high spatial and temporal resolution. J. Hydrometeor., 5, 487-503, https://doi.org/ 10.1175/1525-7541(2004)005<0487:CAMTPG >2.0.CO;2.

Kanada, S., H. Tsuguti, T. Kato, and F. Fujibe, 2014: Diurnal variation of precipitation around western Japan during the warm season. SOLA, 10, 72-77, https://doi.org/10.2151/SOLA.2014015 .

Kobayashi, S., and Coauthors, 2015: The JRA-55 reanalysis: General specifications and basic characteristics. J. Meteor. Soc. Japan, 93, 5-48, https://doi.org/10.2151/jmsj.2015-001.

Kodama, Y.-M., 1993: Large-scale common features of subtropical convergence zones (the baiu frontal zone, the SPCZ, and the SACZ). Part II: Conditions of the circulations for generating the STCZs. J. Meteor. Soc. Japan, 71, 581-610, https://doi.org/ 10.2151/jmsj1965.71.5_581.

Laing, A., and J. Fritsch, 1997: The global population of mesoscale convective complexes. Quart. J. Roy. Meteor. Soc., 123, 389405, https://doi.org/10.1002/qj.49712353807.
Li, C., W. Chen, X. Hong, and R. Lu, 2017: Why was the strengthening of rainfall in summer over the Yangtze River valley in 2016 less pronounced than that in 1998 under similar preceding El Niño events?-Role of midlatitude circulation in August. Adv. Atmos. Sci., 34, 1290-1300, https://doi.org/ 10.1007/s00376-017-7003-8.

Luo, Y., Y. Gong, and D. Zhang, 2014: Initiation and organizational modes of an extreme-rain-producing mesoscale convective system along a mei-yu front in East China. Mon. Wea. Rev., 142, 203-221, https://doi.org/10.1175/MWR-D-13-00111.1.

Maddox, R., 1980: Mesoscale convective complexes. Bull. Amer. Meteor. Soc., 61, 1374-1387, https://doi.org/10.1175/15200477(1980)061<1374:MCC >2.0.CO;2.

Ninomiya, K., 1984: Characteristics of baiu front as predominant subtropical front in the summer Northern Hemisphere. J. Meteor. Soc. Japan, 62, 880-894, https://doi.org/10.2151/ jmsj1965.62.6_880.

, 1991: Polar low development over the east coast of the Asian continent on 9-11 December 1985. J. Meteor. Soc. Japan, 69, 669-685, https://doi.org/10.2151/jmsj1965.69.6_669.

, and Y. Shibagaki, 2007: Multi-scale features of the meiyubaiu front and associated precipitation systems. J. Meteor. Soc. Japan, 85B, 103-122, https://doi.org/10.2151/jmsj.85B.103.

Ren, X., X. Yang, and X. Sun, 2013: Zonal oscillation of western Pacific subtropical high and subseasonal SST variations during Yangtze persistent heavy rainfall events. J. Climate, 26, 89298946, https://doi.org/10.1175/JCLI-D-12-00861.1.

Reynolds, R., T. Smith, C. Liu, D. Chelton, K. Casey, and M. Schlax, 2007: Daily high-resolution-blended analysis for sea surface temperatures. J. Climate, 20, 5473-5496, https:// doi.org/10.1175/2007JCLI1824.1.

Sampe, T., and S. Xie, 2010: Large-scale dynamics of the meiyu-baiu rainband: Environmental forcing by the westerly jet. J. Climate, 23, 113-134, https://doi.org/10.1175/2009JCLI3128.1.

Shin, U., T.-Y. Lee, and S.-H. Park, 2019: Environment and processes for heavy rainfall in the early morning over the Korean peninsula during episodes of cloud clusters associated with mesoscale troughs. J. Meteor. Soc. Japan, 97, 633-655, https:// doi.org/10.2151/jmsj.2019-036.

Shinoda, T., H. Uyeda, and K. Yoshimura, 2005: Structure of moist layer and sources of water over the southern region far from the meiyu/baiu front. J. Meteor. Soc. Japan, 83, 137-152, https:// doi.org/10.2151/jmsj.83.137.

Sun, J., and F. Zhang, 2012: Impacts of mountain-plains solenoid on diurnal variations of rainfall along the mei-yu front over the East China plains. Mon. Wea. Rev., 140,379-397, https://doi.org/ 10.1175/MWR-D-11-00041.1.

Tang, Y., J. Gan, L. Zhao, and K. Gao, 2006: On the climatology of persistent heavy rainfall events in China. Adv. Atmos. Sci., 23, 678-692, https://doi.org/10.1007/s00376-006-0678-x.

Trier, S., C. Davis, D. Ahijevych, M. Weisman, and G. Bryan, 2006: Mechanisms supporting long-lived episodes of propagating nocturnal convection within a 7-day WRF model simulation. J. Atmos. Sci., 63, 2437-2461, https://doi.org/10.1175/ JAS3768.1.

—_ - - and R. Carbone, 2014: Mechanisms governing the persistence and diurnal cycle of a heavy rainfall corridor. J. Atmos. Sci., 71, 4102-4126, https://doi.org/10.1175/JAS-D14-0134.1.

, J. Wilson, D. Ahijevych, and R. Sobash, 2017: Mesoscale vertical motions near nocturnal convection initiation in PECAN. Mon. Wea. Rev., 145, 2919-2941, https://doi.org/10.1175/MWRD-17-0005.1. 
Tuttle, J., and C. Davis, 2006: Corridors of warm season precipitation in the central United States. Mon. Wea. Rev., 134, 22972317, https://doi.org/10.1175/MWR3188.1.

Wallace, J., 1975: Diurnal variations in precipitation and thunderstorm frequency over the conterminous United States. Mon. Wea. Rev., 103, 406-419, https://doi.org/10.1175/15200493(1975)103<0406:DVIPAT > 2.0.CO;2.

Wang, B., R. Wu, and X. Fu, 2000: Pacific-East Asian teleconnection: How does ENSO affect East Asian climate? J. Climate, 13, 1517-1536, https://doi.org/10.1175/1520-0442(2000)013<1517: PEATHD $>2.0 . \mathrm{CO} ; 2$.

Wang, C.-C., G. T.-J. Chen, and R. Carbone, 2004: A climatology of warm-season cloud patterns over East Asia based on GMS infrared brightness temperature observations. Mon. Wea. Rev., 132, 1606-1629, https://doi.org/10.1175/1520-0493(2004) 132<1606:ACOWCP>2.0.CO;2.

— J. C.-S. Hsu, G. T.-J. Chen, and D.-I. Lee, 2014: A study of two propagating heavy-rainfall episodes near Taiwan during SoWMEX/TiMREX IOP-8 in June 2008. Part II: Sensitivity tests on the roles of synoptic conditions and topographic effects. Mon. Wea. Rev., 142, 2644-2664, https://doi.org/10.1175/ MWR-D-13-00330.1.

Wang, W.-C., W. Gong, and H. Wei, 2000: A regional model simulation of the 1991 severe precipitation event over the YangtzeHuai River valley. Part I: Precipitation and circulation statistics. J. Climate, 13, 74-92, https://doi.org/10.1175/1520-0442(2000) 013<0074:ARMSOT>2.0.CO;2.

Wang, Y., 1992: Effects of blocking anticyclones in Eurasia in the rainy season (meiyu/baiu season). J. Meteor. Soc. Japan, 70, 929-951, https://doi.org/10.2151/jmsj1965.70.5_929.

Wei, W., R. Zhang, M. Wen, and S. Yang, 2017: Relationship between the Asian westerly jet stream and summer rainfall over central Asia and north China: Roles of the Indian monsoon and the South Asian high. J. Climate, 30, 537-552, https:// doi.org/10.1175/JCLI-D-15-0814.1.

Wu, Y., and Coauthors, 2018: Diurnal variations of summer precipitation over the regions east to Tibetan Plateau. Climate Dyn., 51, 4287-4307, https://doi.org/10.1007/s00382-017-4042-x.

Xie, S.-P., K. Hu, J. Hafner, H. Tokinaga, Y. Du, G. Huang, and T. Sampe, 2009: Indian Ocean capacitor effect on Indo-western Pacific climate during the summer following El Niño. J. Climate, 22, 730-747, https://doi.org/10.1175/2008JCLI2544.1.

_- Y. Kosaka, Y. Du, K. Hu, J. Chowdary, and G. Huang, 2016: Indo-western Pacific Ocean capacitor and coherent climate anomalies in post-ENSO summer: A review. Adv. Atmos. Sci., 33, 411-432, https://doi.org/10.1007/s00376-015-5192-6.

$\mathrm{Xu}$, W., and E. Zipser, 2011: Diurnal variations of precipitation, deep convection, and lightning over and east of the eastern Tibetan Plateau. J. Climate, 24, 448-465, https://doi.org/10.1175/ 2010JCLI3719.1.

Xue, M., X. Luo, K. Zhu, Z. Sun, and J. Fei, 2018: The controlling role of boundary layer inertial oscillations in meiyu frontal precipitation and its diurnal cycles over China. J. Geophys. Res. Atmos., 123, 5090-5115, https://doi.org/10.1029/2018JD028368.

Yamada, H., B. Geng, H. Uyeda, and K. Tsuboki, 2007a: Thermodynamic impact of the heated landmass on the nocturnal evolution of a cloud cluster over a meiyu-baiu front. J. Meteor. Soc. Japan, 85, 663-685, https://doi.org/10.2151/ jmsj.85.663.

,,--- , and,$- 2007 \mathrm{~b}$ : Role of the heated landmass on the evolution and duration of a heavy rain episode over a meiyu-baiu frontal zone. J. Meteor. Soc. Japan, 85, 687-709, https://doi.org/10.2151/jmsj.85.687.

Yamazaki, N., and T.-C. Chen, 1993: Analysis of the East Asian monsoon during early summer of 1979: Structure of the baiu front and its relationship to large-scale fields. J. Meteor. Soc. Japan, 71, 339-355, https://doi.org/10.2151/jmsj1965.71.3_339.

Yang, J., B. Wang, B. Wang, and Q. Bao, 2010: Biweekly and 21-30-day variations of the subtropical summer monsoon rainfall over the lower reach of the Yangtze River basin. J. Climate, 23, 1146-1159, https://doi.org/10.1175/2009JCLI3005.1.

Yang, X., J. Fei, X. Huang, X. Cheng, L. Carvalho, and H. He, 2015: Characteristics of mesoscale convective systems over China and its vicinity using geostationary satellite FY2. J. Climate, 28, 4890-4907, https://doi.org/10.1175/JCLI-D-14-00491.1.

Yin, S., D. Chen, and Y. Xie, 2009: Diurnal variations of precipitation during the warm season over China. Int. J. Climatol., 29, 1154-1170, https://doi.org/10.1002/joc.1758.

Yu, R., Y. Xu, T. Zhou, and J. Li, 2007: Relation between rainfall duration and diurnal variation in the warm season precipitation over central eastern China. Geophys. Res. Lett., 34, L13703, https://doi.org/10.1029/2007GL030315.

_ J. Li, H. Chen, and W. Yuan, 2014: Progress in studies of the precipitation diurnal variation over contiguous China. J. Meteor. Res., 28, 877-902, https://doi.org/10.1007/s13351-014-3272-7.

Yuan, W., R. Yu, H. Chen, J. Li, and M. Zhang, 2010: Subseasonal characteristics of diurnal variation in summer monsoon rainfall over central eastern China. J. Climate, 23, 6684-6695, https://doi.org/10.1175/2010JCLI3805.1.

,-- , and J. Li, 2013: Changes in the diurnal cycles of precipitation over eastern China in the past 40 years. Adv. Atmos. Sci., 30, 461-467, https://doi.org/10.1007/s00376-012-2092-x.

Zeng, W., G. Chen, Y. Du, and Z. Wen, 2019: Diurnal variations of low-level winds and rainfall response to large-scale circulations during a heavy rainfall event. Mon. Wea. Rev., 147, 39814004, https://doi.org/10.1175/MWR-D-19-0131.1.

Zhang, Y., J. Sun, and S. Fu, 2014a: Impacts of diurnal variation of mountain-plain solenoid circulations on precipitation and vortices east of the Tibetan Plateau during the mei-yu season. Adv. Atmos. Sci., 31, 139-153, https://doi.org/10.1007/s00376013-2052-0.

— , F. Zhang, and J. Sun, 2014b: Comparison of the diurnal variations of warm-season precipitation for East Asia vs. North America downstream of the Tibetan Plateau vs. the Rocky Mountains. Atmos. Chem. Phys., 14, 10 741-10 759, https://doi.org/10.5194/acp-14-10741-2014.

,-- C. Davis, and J. Sun, 2018: Diurnal evolution and structure of long-lived mesoscale convective vortices along the mei-yu front over the East China plains. J. Atmos. Sci., 75, 1005-1025, https://doi.org/10.1175/JAS-D-17-0197.1.

Zhang, Y., M. Xue, K. Zhu, and B. Zhou, 2019: What is the main cause of diurnal variation and nocturnal peak of summer precipitation in Sichuan Basin, China? The key role of boundary layer low-level jet inertial oscillations. J. Geophys. Res. Atmos., 124, 2643-2664, https://doi.org/10.1029/ 2018JD029834.

Zhou, T., R. Yu, H. Chen, A. Dai, and Y. Pan, 2008: Summer precipitation frequency, intensity, and diurnal cycle over China: A comparison of satellite data with rain gauge observations. J. Climate, 21, 3997-4010, https://doi.org/10.1175/ 2008JCLI2028.1. 